\title{
Relativistic Klein-Gordon-Maxwell multistream model for quantum plasmas
}

\author{
F. Haas \\ Departamento de Física, Universidade Federal do Paraná, 81531-990 Curitiba, Paraná, Brazil
}

B. Eliasson

International Centre for Advanced Studies in Physical Sciences and Institute for Theoretical Physics, Faculty of Physics \& Astronomy, Ruhr University Bochum, D-44780 Bochum, Germany

\begin{abstract}
P. K. Shukla
International Centre for Advanced Studies in Physical Sciences and Institute for Theoretical Physics, Faculty of Physics \& Astronomy, Ruhr University Bochum, D-44780 Bochum, Germany and Department of Mechanical and Aerospace Engineering \& Center for Energy Research, University of California San Diego, La Jolla, California 92093, USA
\end{abstract}

(Received 19 February 2012; published 18 May 2012)

\begin{abstract}
A multistream model for spinless electrons in a relativistic quantum plasma is introduced by means of a suitable fluidlike version of the Klein-Gordon-Maxwell system. The one- and two-stream cases are treated in detail. A new linear instability condition for two-stream quantum plasmas is obtained, generalizing the previously known nonrelativistic results. In both the one- and two-stream cases, steady-state solutions reduce the model to a set of coupled nonlinear ordinary differential equations, which can be numerically solved, yielding a manifold of nonlinear periodic and soliton structures. The validity conditions for the applicability of the model are addressed.
\end{abstract}

DOI: 10.1103/PhysRevE.85.056411

PACS number(s): 52.27.Ny, 52.35.Qz, 52.35.Sb

\section{INTRODUCTION}

The interest in relativistic quantum plasma systems is growing exponentially, not only because of the relevance to astrophysical problems but also due to the fast advances in strong laser-solid plasma interaction experiments. Indeed, the development of multi-Peta-Watt lasers will soon make it possible to address simultaneous quantum and relativistic effects in laboratory plasmas [1]. Relativistic quantum kinetic models have been proposed in the treatment of the plasma dispersion function for a Fermi-Dirac equilibrium [2] and in the study of relativistic effects for quantum ion-acoustic wave propagation [3]. Also, a covariant Wigner function theory for relativistic quantum plasmas described by the Dirac-Maxwell system has been suggested [4], as well as the spinless (KleinGordon-Maxwell) analog has been presented [5]. The KleinGordon-Maxwell system of equations has been applied to the analysis of parametric scattering instabilities in relativistic laser-quantum plasma interactions [6], while recent models have been introduced based on the Dirac-Maxwell equations describing the propagation of light in Dirac matter [7]. Moreover, the response of relativistic degenerate plasmas [8] and the quasiclassical relativistic Bargmann-Michel-Telegdi model for the spin dependence in a magnetized electron gas [9] have been addressed.

Relativistic two-stream instabilities are traditionally known to be important for the electron heating in intense laser-plasma interaction experiments [10], as well as in astrophysical relativistic shocks [11], and could be important for pulsar glitches $[12,13]$, where superfluid neutrons and superconducting protons coexist with relativistic electrons [14]. In addition, the two-stream instabilities between electrons and/or holes are also believed to exist in semiconductor plasmas [15-17].

In a lower level of approximation than kinetic theory, quantum plasma hydrodynamic models are popular tools (see, e.g., Refs. [18-21]), since they allow an efficient treatment of nonlinear phenomena, from both the analytical and numerical viewpoints. Starting from the Dirac-Maxwell system, there are hydrodynamic models for relativistic quantum plasmas [22], which have been extended to incorporate particle-antiparticle effects in the wave propagation [23], as well as relativistic quantum corrections to laser wakefield acceleration [24]. In these fluid formulations, we note, in particular, the nontrivial form of the relativistic extension of the quantum tunneling force in the momentum transport equation. Electromagnetic quantum hydrodynamic wave equations have also been considered including relativistically degenerate electron fluids [25], based on previous spin-1/2 hydrodynamic models [26-29]. We note that hydrodynamic versions of the Klein-GordonMaxwell system have been used in the past [30] and recently in the context of laser physics [31].

In this paper, we introduce a relativistic multistream quantum plasma model starting from the Klein-Gordon-Maxwell system of equations. We adapt the formalism according to the classical [32] and quantum [33] multistream model for plasmas and show its usefulness in a paradigmatic plasma problem, namely, the linear and nonlinear features of the quantum two-stream instability in the relativistic regime. The simplicity of the Klein-Gordon equations, in comparison to the Dirac equation, makes it a natural candidate for the extension of nonrelativistic quantum plasma theories to the relativistic regime, when electron spin- $1 / 2$ effects can be neglected. Therefore, a direct comparison to existing results on quantum plasmas can be obtained in an easier way. Moreover, a hydrodynamic formulation based on the Klein-Gordon-Maxwell system of equations strongly favors the development of new analytical and numerical tools for relativistic quantum plasmas. On the other hand, the domain of applicability is restricted to plasmas where the electron spin-1/2 effects are not decisive, like in close to isotropic equilibrium configurations and in the study of the propagation of high frequency electromagnetic waves, since the spin contribution is expected to be more important for low frequency waves [5]. On the same footing, spinspin interactions which may give rise, e.g., to ferromagnetic 
behavior [34] are not included either. For the parameters in this work, we have no quantized electromagnetic fields, so that quantum field theoretic results involving, e.g., pair creation are not included. Streaming instabilities in nonrelativistic quantum plasmas are attracting considerable interest, since they display many surprising characteristics of pure quantum origin. Among these, we have a new instability branch for large wave numbers, as well as new nonlinear spatially periodic solutions in the steady-state case [35]. Besides formulating the relativistic version of the quantum multistream model for plasmas, the purpose of the present work is to extend the analysis of the quantum two-stream instability to the relativistic case.

The manuscript is organized as follows. In Sec. II we introduce the multistream Klein-Gordon-Maxwell system of equations, casting it into a suitable fluidlike formulation. The one-stream case is treated in detail in Sec. III, where linear wave propagation is studied considering small amplitude perturbations around homogeneous equilibria, and a rich variety of nonlinear periodic as well as soliton structures are found numerically. An existence criterion for solitary wave solutions is obtained. In Sec. IV, the relativistic quantum two-stream case is studied in depth. The linear relativistic quantum two-stream instability problem is fully characterized. In this regard, a main result of this work is the derivation of the instability condition in Eq. (82), which provides a natural generalization to the nonrelativistic instability criterion [33]. Both nonlinear periodic and soliton structures are found numerically, and an existence criterion for localized solutions is obtained theoretically. Section V is dedicated to the final conclusions, including a detailed account of the validity domain of our model equations.

\section{THE KLEIN-GORDON-MAXWELL MULTISTREAM MODEL}

We here consider a relativistic multistream quantum plasma where the electrons are described by a statistical mixture of $N$ pure states, with each wave function $\psi_{j}$ satisfying the KleinGordon equation

$$
\mathcal{W}^{2} \psi_{j}-c^{2} \mathcal{P}^{2} \psi_{j}-m^{2} c^{4} \psi_{j}=0, \quad j=1, \ldots, N,
$$

where we have defined the energy and momentum operators, respectively, as

$$
\mathcal{W}=i \hbar \frac{\partial}{\partial t}+e \phi
$$

and

$$
\mathcal{P}=-i \hbar \nabla+e \mathbf{A}
$$

Here, $m$ and $-e$ are the electron mass and charge, respectively, $\hbar$ is the Planck constant divided by $2 \pi, c$ is the speed of light in vacuum, and $\phi$ and $\mathbf{A}$ are the scalar and vector potentials, respectively.

The electric charge and current densities are, respectively,

$$
\rho=-\frac{e}{2 m c^{2}} \sum_{j=1}^{N}\left[\psi_{j}^{*} \mathcal{W} \psi_{j}+\psi_{j}\left(\mathcal{W} \psi_{j}\right)^{*}\right]
$$
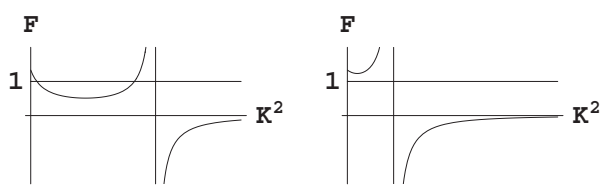

FIG. 1. Left: A typical case of stable linear one-stream oscillations when $F_{\min }<1$. Right: A typical unstable case where $F_{\min }>1$. The pole is at $K^{2}=4 p^{2} / H^{2}$.

and

$$
\mathbf{J}=-\frac{e}{2 m} \sum_{j=1}^{N}\left[\psi_{j}^{*} \mathcal{P} \psi_{j}+\psi_{j}\left(\mathcal{P} \psi_{j}\right)^{*}\right] .
$$

The charge and current densities fulfill the continuity equation

$$
\frac{\partial \rho}{\partial t}+\nabla \cdot \mathbf{J}=0
$$

The self-consistent scalar and vector potentials are obtained from the inhomogeneous Maxwell's equations, using the Coulomb gauge $\boldsymbol{\nabla} \cdot \mathbf{A}=0$, as

$$
\begin{aligned}
& \nabla^{2} \phi=-\frac{1}{\varepsilon_{0}}\left(\rho+n_{0} e\right), \\
& \square \mathbf{A}=\mu_{0} \mathbf{J}-\frac{1}{c^{2}} \nabla \frac{\partial \phi}{\partial t},
\end{aligned}
$$

where a fixed neutralizing ion background of the charge density $e n_{0}$ was added, and where $\varepsilon_{0}$ and $\mu_{0}$ denote the vacuum electric permittivity and magnetic permeability, respectively. Here, the $\mathrm{d}$ 'Alembert operator is

$$
=\frac{1}{c^{2}} \frac{\partial^{2}}{\partial t^{2}}-\nabla^{2}
$$

The resulting Klein-Gordon-Maxwell system of Eqs. (1), (7), and (8) describes the nonlinear interactions in relativistic quantum plasmas where spin effects are negligible. In explicit form, the Klein-Gordon equation reads

$$
\begin{gathered}
\square \psi_{j}-\frac{i e}{\hbar c^{2}}\left(\frac{\partial \phi}{\partial t} \psi_{j}+2 \phi \frac{\partial \psi_{j}}{\partial t}+2 c^{2} \mathbf{A} \cdot \nabla \psi_{j}\right) \\
+\frac{1}{\hbar^{2}}\left(e^{2} A^{2}-\frac{e^{2} \phi^{2}}{c^{2}}+m^{2} c^{2}\right) \psi_{j}=0 .
\end{gathered}
$$

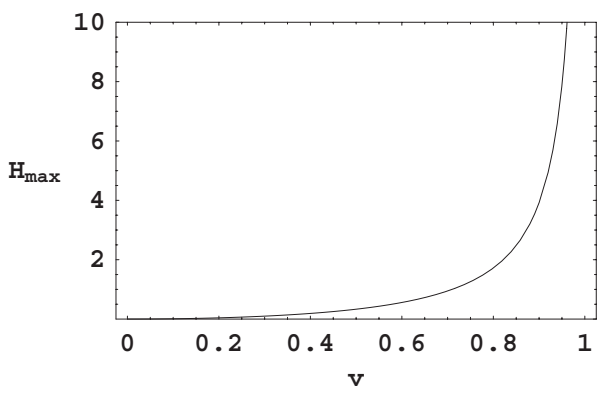

FIG. 2. Maximum quantum parameter $H_{\max }$ in Eq. (55) as a function of the velocity $v$ measured in units of $c$. 

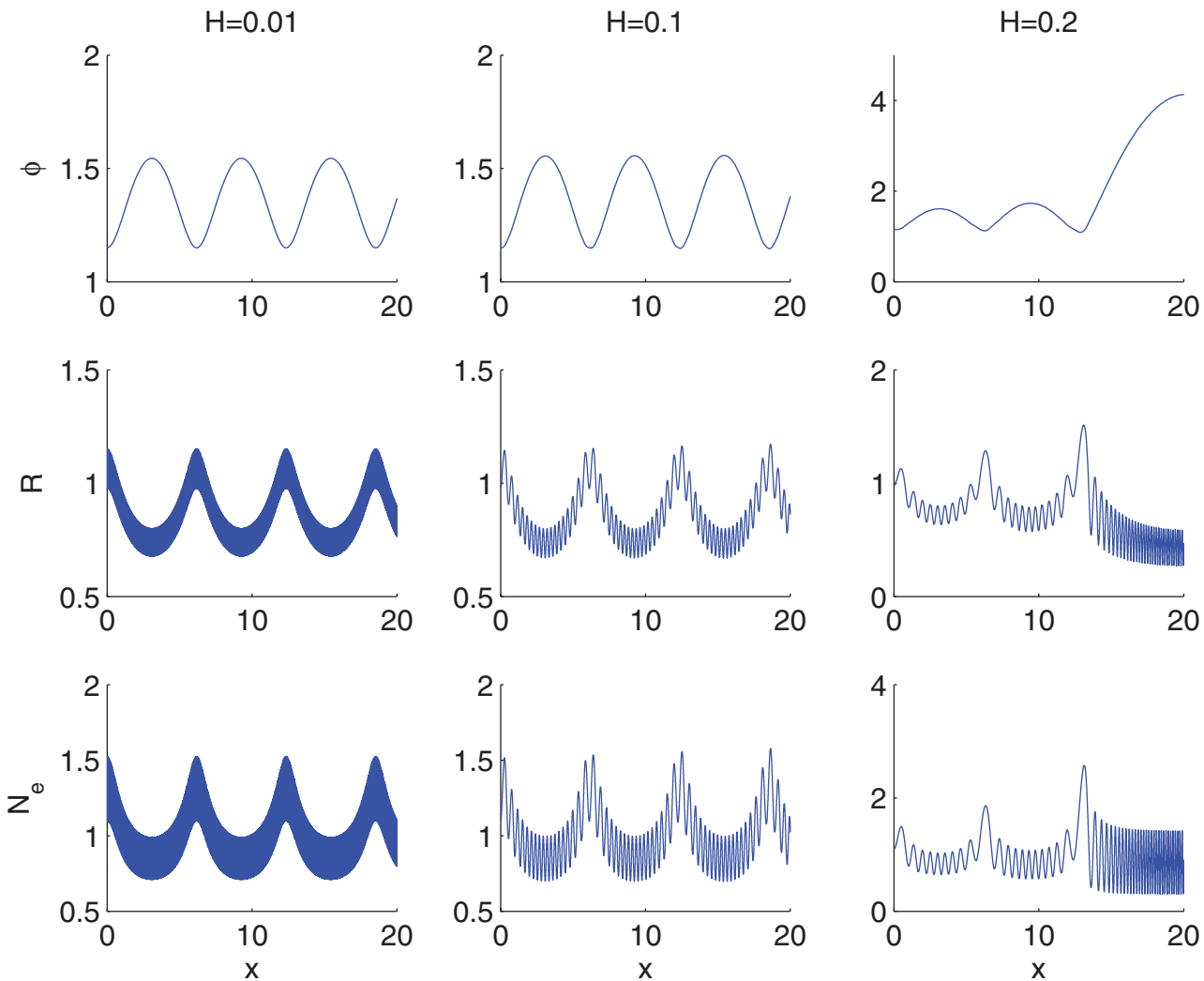

FIG. 3. (Color online) Spatial profiles of $\phi, R$, and the electron number density $N_{e}=R^{2} \phi$ (top to bottom panels) for $\gamma=1.3$ and $H=0.01$ (left column), $H=0.1$ (middle column), and $H=0.2$ (right column). The solution was set to $\phi(0)=1.15, R(0)=0.98$, and $\phi^{\prime}(0)=R^{\prime}(0)=0$ at the left boundary.

Following Takabayasi [30], it is convenient to introduce a fluidlike formulation in terms of the eikonal decomposition

$$
\psi_{j}=R_{j} \exp \left(i S_{j} / \hbar\right),
$$

where the amplitude $R_{j}$ and the phase $S_{j}$ are real functions. Separating the real and imaginary parts of the Klein-Gordon equation, we have

$\frac{1}{c^{2}}\left(\frac{\partial S_{j}}{\partial t}-e \phi\right)^{2}-\left(\nabla S_{j}+e \mathbf{A}\right)^{2}-m^{2} c^{2}=\frac{\hbar^{2} \square R_{j}}{R_{j}}$

and

$$
\begin{gathered}
R_{j}\left(\square S_{j}-\frac{e}{c^{2}} \frac{\partial \phi}{\partial t}\right)+\frac{2}{c^{2}} \frac{\partial R_{j}}{\partial t}\left(\frac{\partial S_{j}}{\partial t}-e \phi\right) \\
-2 \nabla R_{j} \cdot\left(\nabla S_{j}+e \mathbf{A}\right)=0 .
\end{gathered}
$$

In terms of $R_{j}$ and $S_{j}$, the charge and current densities are, respectively,

$$
\rho=\frac{e}{m c^{2}} \sum_{j=1}^{N} R_{j}^{2}\left(\frac{\partial S_{j}}{\partial t}-e \phi\right)
$$

and

$$
\mathbf{J}=-\frac{e}{m} \sum_{j=1}^{N} R_{j}^{2}\left(\nabla S_{j}+e \mathbf{A}\right) .
$$

Alternative hydrodynamiclike methods are also available, such as, for instance, the Feshbach-Villars formalism [36] where initially the Klein-Gordon equation is split into a pair of first order in time partial differential equations. However, the resulting set of equations turns out to appear much more involved than in the present Takabayasi approach, which we use due to its formal simplicity.

From now on we concentrate on the electrostatic case and assume $\mathbf{A}=0$. In this situation, the relevant equations are

$$
\begin{gathered}
\frac{1}{c^{2}}\left(\frac{\partial S_{j}}{\partial t}-e \phi\right)^{2}-\left(\nabla S_{j}\right)^{2}-m^{2} c^{2}=\frac{\hbar^{2} \square R_{j}}{R_{j}}, \\
R_{j}\left(\square S_{j}-\frac{e}{c^{2}} \frac{\partial \phi}{\partial t}\right)+\frac{2}{c^{2}} \frac{\partial R_{j}}{\partial t}\left(\frac{\partial S_{j}}{\partial t}-e \phi\right) \\
-2 \nabla R_{j} \cdot \nabla S_{j}=0,
\end{gathered}
$$

and

$$
\nabla^{2} \phi=-\frac{e}{\varepsilon_{0}}\left[\sum_{j=1}^{N} \frac{R_{j}^{2}}{m c^{2}}\left(\frac{\partial S_{j}}{\partial t}-e \phi\right)+n_{0}\right] .
$$

The assumption $\mathbf{A}=0$ is valid as long as the electric current is curl free, $\nabla \times \mathbf{J}=0$. This is true for longitudinal waves in a stationary plasma and parallel to a plasma beam.

\section{ONE-STREAM CASE}

We proceed next to study linear and nonlinear waves for the one-stream case $(N=1)$. For this case the sum in Eq. (18) 

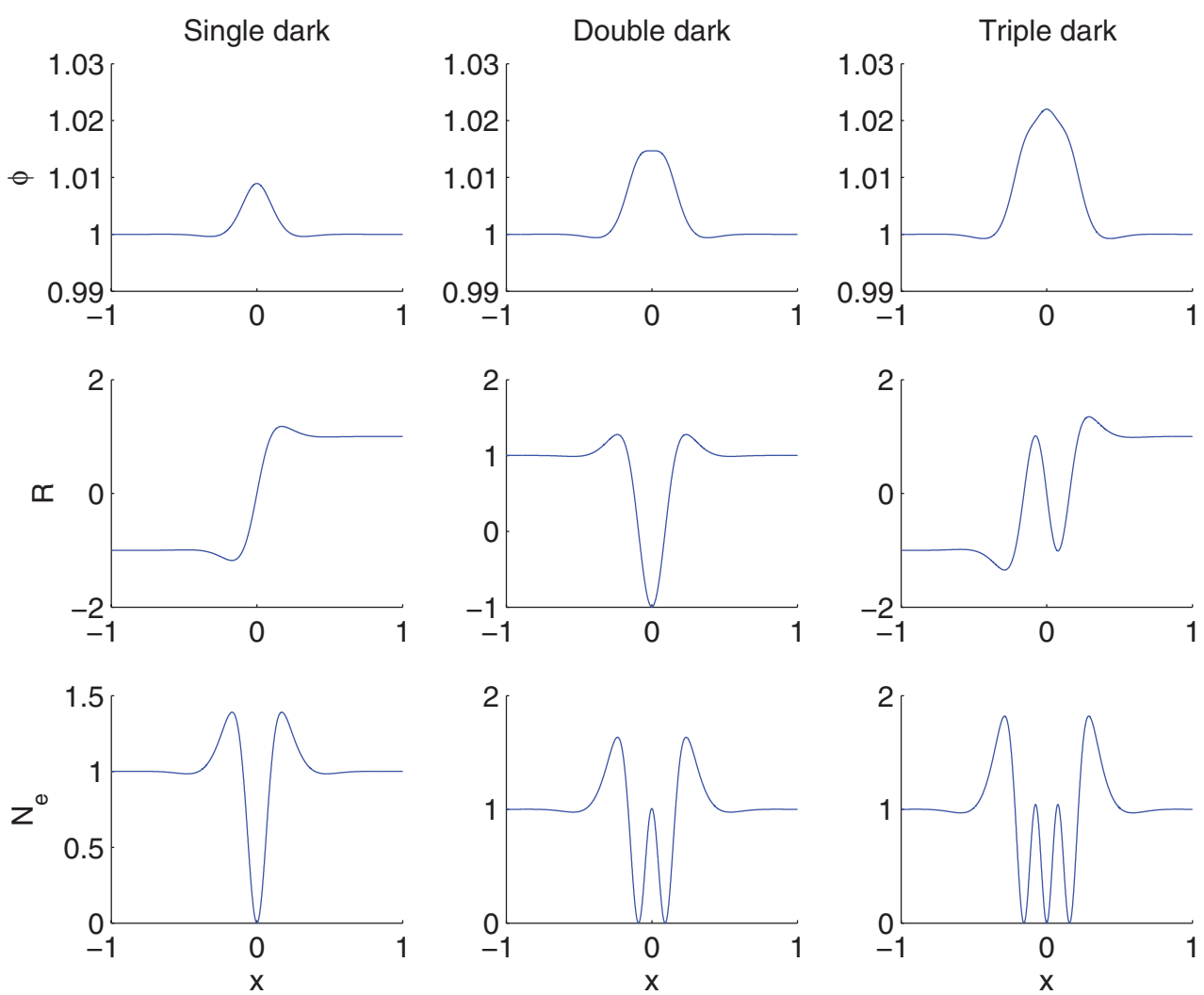

FIG. 4. (Color online) Spatial profiles of $\phi, R$, and the electron density $N_{e}=R^{2} \phi$ (top to bottom panels), showing single, double, and triple dark solitary waves (left to right columns) for the zero beam speed case $v=0$ with $H=0.01$. The solution is set to $\phi=|R|=1$ at the left and right boundaries ( $R=-1$ on the left boundary for the single and triple dark solitons).

collapses to one term involving $R_{1}=R$ and $S_{1}=S$, and Eqs. (16)-(18) become

$$
\begin{gathered}
\frac{1}{c^{2}}\left(\frac{\partial S}{\partial t}-e \phi\right)^{2}-(\nabla S)^{2}-m^{2} c^{2}=\frac{\hbar^{2} \square R}{R}, \\
R\left(\square S-\frac{e}{c^{2}} \frac{\partial \phi}{\partial t}\right)+\frac{2}{c^{2}} \frac{\partial R}{\partial t}\left(\frac{\partial S}{\partial t}-e \phi\right)-2 \nabla R \cdot \nabla S=0,
\end{gathered}
$$

and

$$
\nabla^{2} \phi=-\frac{e}{\varepsilon_{0}}\left[\frac{R^{2}}{m c^{2}}\left(\frac{\partial S}{\partial t}-e \phi\right)+n_{0}\right] .
$$

\section{A. Linear waves}

The system of Eqs. (19)-(21) has the equilibrium solution

$$
R=\sqrt{\frac{n_{0}}{\gamma}}, \quad S=-\gamma m c^{2} t+\mathbf{p} \cdot \mathbf{r}, \quad \phi=0,
$$

where

$$
\gamma=\left(1+\frac{p^{2}}{m^{2} c^{2}}\right)^{1 / 2}
$$

is the relativistic $\gamma$ factor for a beam momentum p. By linearizing and assuming perturbations $\sim \exp (i[\mathbf{K} \cdot \mathbf{r}-\Omega t])$, where, for simplicity, we take $\mathbf{K} \| \mathbf{p}$, we obtain

$$
\begin{aligned}
(\Omega-K v)^{2}= & \frac{\omega_{p}^{2}}{\gamma^{3}}+\frac{\hbar^{2}}{4 \gamma^{2} m^{2}}\left(K^{2}-\frac{\Omega^{2}}{c^{2}}\right)^{2} \\
& +\frac{\hbar^{2} \omega_{p}^{2}}{4 \gamma^{3} m^{2} c^{2}}\left(K^{2}-\frac{\Omega^{2}}{c^{2}}\right),
\end{aligned}
$$

where $v=p /(\gamma m)$ is the equilibrium beam speed and $\omega_{p}=$ $\left[n_{0} e^{2} /\left(m \varepsilon_{0}\right)\right]^{1 / 2}$.

In the nonstreaming limit $p \rightarrow 0$ (and $\gamma=1$ ), Eq. (24) is identical to Eq. (4.21) of Kowalenko et al. [37]. On the other hand, relativistic effects are noticeable for large beam velocity so that $\gamma$ is appreciably larger than unity.

For the general one-stream case with waves propagating obliquely to the beam direction, the assumption $\mathbf{A}=0$ fails, and one has to involve the full set of Maxwell's equations. However, in the one-stream case, one can start with the threedimensional dispersion relation in the beam frame and then Lorentz transform the result to the laboratory frame. In the beam frame, the dispersion relation is

$$
\begin{aligned}
\left(\Omega^{\prime}\right)^{2}= & \left(\omega_{p}^{\prime}\right)^{2}+\frac{\hbar^{2}}{4 m^{2}}\left[\left(K^{\prime}\right)^{2}-\frac{\left(\Omega^{\prime}\right)^{2}}{c^{2}}\right]^{2} \\
& +\frac{\hbar^{2}\left(\omega_{p}^{\prime}\right)^{2}}{4 m^{2} c^{2}}\left[\left(K^{\prime}\right)^{2}-\frac{\left(\Omega^{\prime}\right)^{2}}{c^{2}}\right],
\end{aligned}
$$



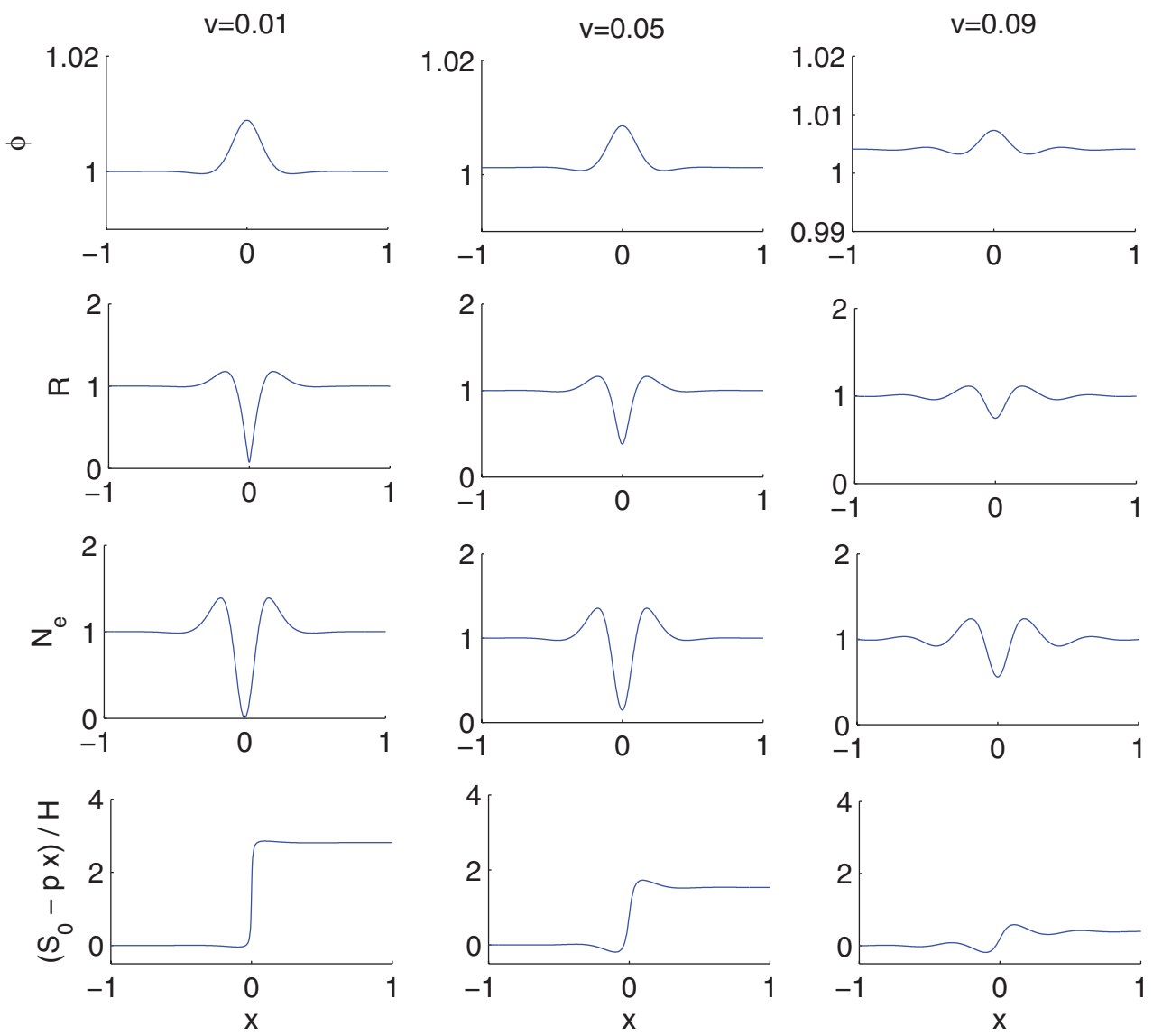

FIG. 5. (Color online) Spatial profiles of $\phi, R, N_{e}=R^{2} \phi$, and $\left(S_{0}-p x\right) / H$ (top to bottom panels), for $H=0.01$ and $v=0.01$ (left column), $v=0.05$ (middle column), and $v=0.09$ (right column). The solution was set to $\phi=\gamma$ and $R=1 / \sqrt{\gamma}$ at the left and right boundaries. We see gray solitons with nonzero electron density in the center. The bottom panels show the phase shift $\left(S_{0}-p x\right) / H$ of the wave function.

where the primed $\Omega^{\prime}$ and $\mathbf{K}^{\prime}$ are the angular frequency and the wave vector of the plasma oscillations in the beam frame.

To go from the beam frame to the laboratory frame, we assume for simplicity that the beam velocity is along the $z$ axis. Then, the time and space variables are Lorentz transformed as $t^{\prime}=\gamma\left(t-v z / c^{2}\right), x^{\prime}=x, y^{\prime}=y$, and $z^{\prime}=$ $\gamma(z-v t)$. The corresponding frequency and wave number transformations are $\Omega^{\prime}=\gamma\left(\Omega-v K_{z}\right), K_{x}^{\prime}=K_{x}, K_{y}^{\prime}=K_{y}$, and $K_{z}^{\prime}=\gamma\left(K_{z}-v \Omega / c^{2}\right)$.

The plasma frequency is transformed as $\omega_{p}^{\prime}=\omega_{p} / \sqrt{\gamma}$. One easily verifies that the expression $\left(K^{\prime}\right)^{2}-\left(\Omega^{\prime}\right)^{2} / c^{2}=$ $K^{2}-\Omega^{2} / c^{2}$ is Lorentz invariant. This yields immediately the general dispersion relation for beam oscillations in the laboratory frame,

$$
\begin{aligned}
\left(\Omega-v K_{z}\right)^{2}= & \frac{\omega_{p}^{2}}{\gamma^{3}}+\frac{\hbar^{2}}{4 \gamma^{2} m^{2}}\left(K^{2}-\frac{\Omega^{2}}{c^{2}}\right)^{2} \\
& +\frac{\hbar^{2} \omega_{p}^{2}}{4 \gamma^{3} m^{2} c^{2}}\left(K^{2}-\frac{\Omega^{2}}{c^{2}}\right) .
\end{aligned}
$$

In the formal classical limit $(\hbar=0)$, we have from Eq. (24) the Doppler-shifted relativistic plasma oscillations [38,39] $\Omega=K v+\omega_{p} \gamma^{-3 / 2}$. Using the limit $\Omega \approx K v$ and
$\Omega \gg \omega_{p}$ in the right-hand side of Eq. (24) one obtains

$$
(\Omega-K v)^{2}=\frac{\omega_{p}^{2}}{\gamma^{3}}+\frac{\hbar^{2} K^{4}}{4 \gamma^{6} m^{2}},
$$

which is similar to the expression used by Serbeto et al. [40] in the context of quantum free-electron lasers, and where the last term in the right-hand side can be considered a quantum correction to the relativistic beam-plasma mode.

On the other hand, in the nonrelativistic limit $c \rightarrow \infty$ we have $\gamma=1$ and the familiar result [33]

$$
(\Omega-K v)^{2}=\omega_{p}^{2}+\frac{\hbar^{2} K^{4}}{4 m^{2}},
$$

describing Doppler-shifted quantum Langmuir waves.

Normalizing the physical quantities into dimensionless units according to

$$
\Omega^{*}=\frac{\Omega}{\omega_{p}}, \quad K^{*}=\frac{c K}{\omega_{p}}, \quad p^{*}=\frac{p}{m c}, \quad v^{*}=\frac{v}{c}, \quad H=\frac{\hbar \omega_{p}}{m c^{2}},
$$

one obtains (omitting the asterisks)

$$
(\Omega-K v)^{2}=\frac{1}{\gamma^{3}}+\frac{H^{2}}{4 \gamma^{2}}\left(K^{2}-\Omega^{2}\right)^{2}+\frac{H^{2}}{4 \gamma^{3}}\left(K^{2}-\Omega^{2}\right),
$$



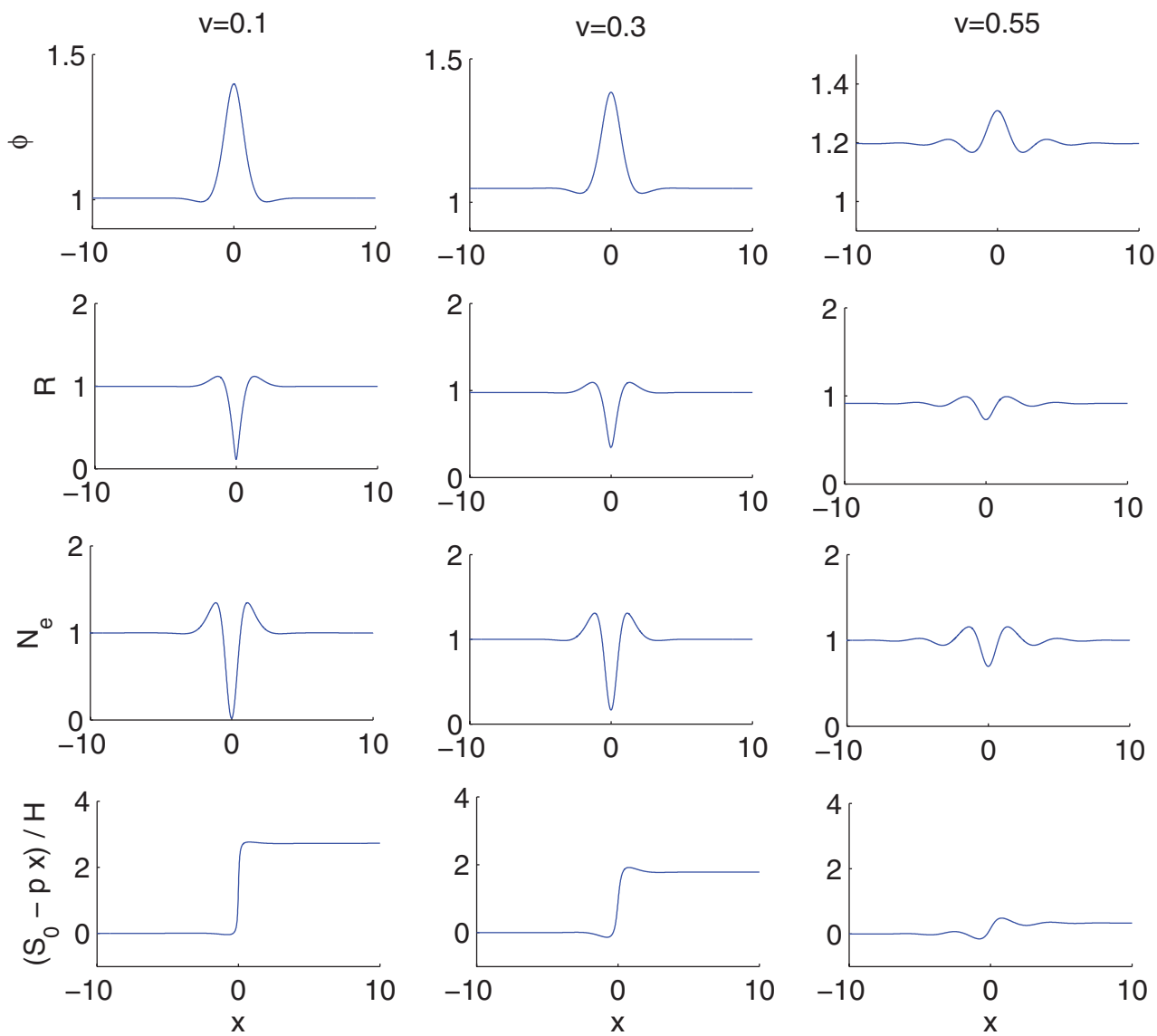

FIG. 6. (Color online) Spatial profiles of $\phi, R, N_{e}=R^{2} \phi$, and $\left(S_{0}-p x\right) / H$ (top to bottom panels), for $H=0.5$ and $v=0.1$ (left column), $v=0.3$ (middle column), and $v=0.55$ (right column). The solution was set to $\phi=\gamma$ and $R=1 / \sqrt{\gamma}$ at the left and right boundaries. We see gray solitons with nonzero electron density in the center. The bottom panels show the phase shift $\left(S_{0}-p x\right) / H$ of the wave function.

where now $\gamma=\left(1+p^{2}\right)^{1 / 2}=\left(1-v^{2}\right)^{-1 / 2}$. If $H \neq 0$ and $v=0$, we solve Eq. (30) to find the modes $\Omega=\Omega_{ \pm}$, with

$\Omega_{ \pm}^{2}=\frac{1}{2}+K^{2}+\frac{2}{H^{2}} \pm 2\left[\frac{K^{2}}{H^{2}}+\left(\frac{1}{4}-\frac{1}{H^{2}}\right)^{2}\right]^{1 / 2}$.

It can be verified that both modes are stable $\left(\Omega_{ \pm}^{2}>0\right)$. It is reasonable to expand the last result assuming a small $H$. Even for laser-compressed matter in the laboratory [41,42], the value of $H$ presently does not significantly exceed $10^{-3}$. On the other hand, for conditions in the interior of white dwarf stars with a quantum coupling parameter exceeding unity we reach the pair creation regime [43], which can be safely treated only within the quantum field theory. The result is

$$
\begin{gathered}
\Omega_{+}^{2}=\frac{4}{H^{2}}+2 K^{2}+\frac{H^{2} K^{2}}{4}\left(1-K^{2}\right)+O\left(H^{4}\right), \\
\Omega_{-}^{2}=1-\frac{H^{2} K^{2}}{4}\left(1-K^{2}\right)+O\left(H^{4}\right) .
\end{gathered}
$$

Here $\Omega_{+}$is the pair branch which goes to infinity as $H \rightarrow 0$, while $\Omega_{-}$is the plasmon mode.

In dimensional units, the pair branch has a cutoff at $\hbar \Omega=2 m c^{2}$. Note that in general $\Omega_{+}^{2}-4 / H^{2}>0$, allowing for particle-antiparticle creation, which is outside the scope of the present model. Nevertheless, the same pair branch turns out to be predicted by more complete, second quantized formulations [37]. On the other hand, the $\Omega_{-}$is actually a backward wave (negative group velocity) for small wave numbers, as mentioned by Kowalenko et al. [37] below their Eq. (4.23).

\section{B. Nonlinear stationary solutions}

Next, we consider nonlinear stationary solutions of Eqs. (19)-(21) in one spatial dimension, of the forms

$$
R=R(x), \quad S=-\gamma m c^{2} t+S_{0}(x), \quad \phi=\phi(x),
$$

so that the original partial differential equation system is converted into a system of ordinary differential equations:

$$
\hbar^{2} c^{2} R^{\prime \prime}=\left[\left(S_{0}^{\prime}\right)^{2} c^{2}-p^{2} c^{2}-2 e \gamma m c^{2} \phi-e^{2} \phi^{2}\right] R,
$$
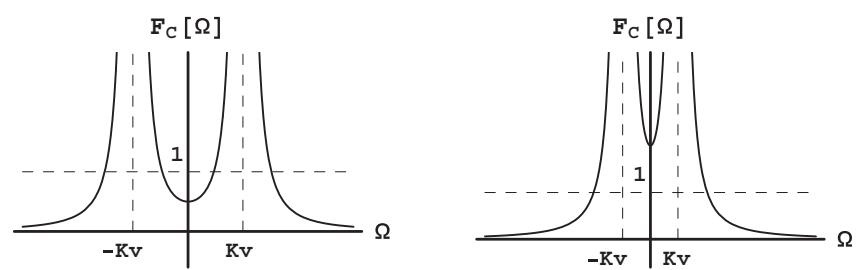

FIG. 7. Left: Stable linear waves satisfying $F_{C}(0)<1$, with the nonquantum characteristic function $F_{C}(\Omega)$ given by Eq. (70). Right: Unstable linear waves. 


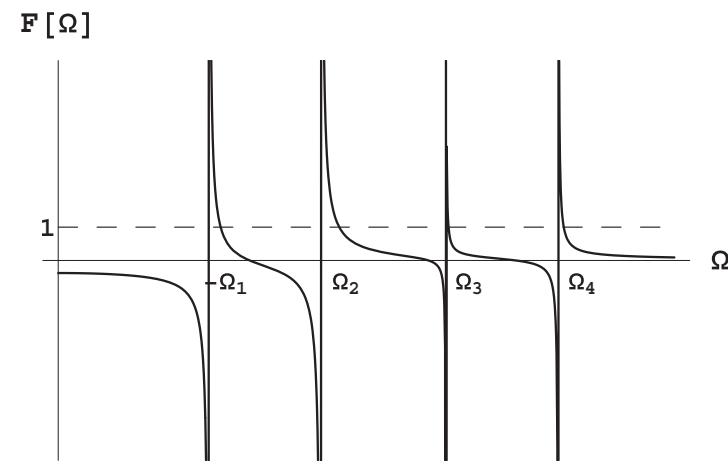

FIG. 8. Generic behavior of the characteristic function $F(\Omega)$ in Eq. (67) for $\hbar K>2 p$. Since $F=1$ at four positive frequencies, the corresponding wave number is stable.

$$
\begin{gathered}
R S_{0}^{\prime \prime}+2 R^{\prime} S_{0}^{\prime}=0, \\
\phi^{\prime \prime}=\frac{e}{\varepsilon_{0}}\left(\gamma R^{2}-n_{0}\right)+\frac{\omega_{p}^{2}}{n_{0} c^{2}} R^{2} \phi,
\end{gathered}
$$

where the primes denote derivatives with respect to $x$.

Equation (36) can be immediately integrated as $R^{2} S_{0}^{\prime}=$ constant. This relation is also equivalent to current continuity $J_{x}=$ constant, which follows from the continuity equation (6) with $\partial \rho / \partial t=0$. Assuming that $R=\sqrt{n_{0} / \gamma}$ and $S_{0}^{\prime}=p$ where the plasma is at equilibrium, we have

$$
R^{2} S_{0}^{\prime}=\frac{n_{0} p}{\gamma} \Rightarrow S_{0}^{\prime}=\frac{n_{0} p}{\gamma R^{2}}
$$

which inserted into Eq. (35) yields

$$
\hbar^{2} c^{2} R^{\prime \prime}+\left(p^{2} c^{2}+2 e \gamma m c^{2} \phi+e^{2} \phi^{2}\right) R=\frac{n_{0}^{2} p^{2} c^{2}}{\gamma^{2} R^{3}} \text {. }
$$

Equations (37) and (39) form a coupled nonlinear system for $\phi$ and $R$, describing steady-state solutions of our relativistic quantum plasma. For nonlinear Schrödinger-type equations, the nonlinear term is focusing or defocusing if it has the same or opposite sign compared to the coefficient in front of the dispersive term. For a focusing nonlinearity, we have the possibility of bright solitons with a localized maximum and a solution going to zero at far away from the soliton, while for a defocusing nonlinearity we have instead dark or gray solitons,

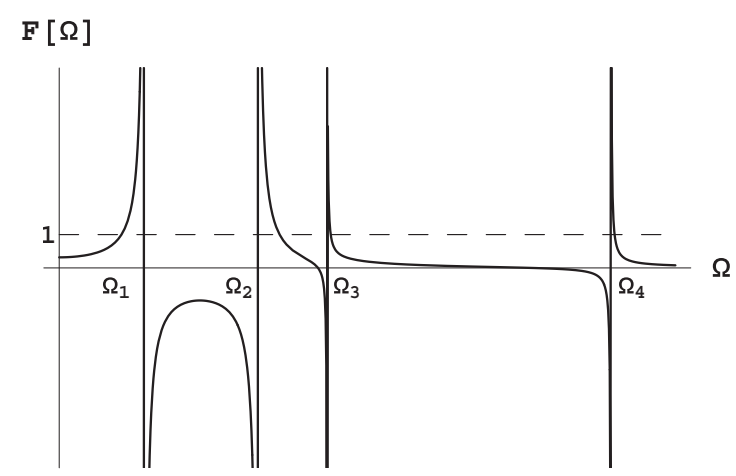

FIG. 9. Generic behavior of the characteristic function $F(\Omega)$ in Eq. (67) for $\hbar K<2 p$, in the stable cases where $F(0)<1$.

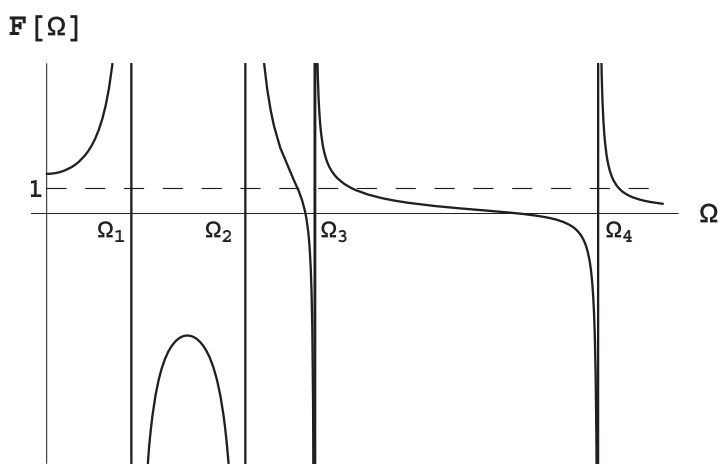

FIG. 10. Generic behavior of the characteristic function $F(\Omega)$ in Eq. (67) for $\hbar K<2 p$, in the unstable cases where $F(0)>1$.

characterized by a local amplitude minimum (going to zero for dark solitons) in the solution, which goes to a finite amplitude far away from the soliton. Here, the nonlinear Schrödinger-like Eq. (39) is coupled nonlinearly with Eq. (37), so the situation is more involved. In general, however, a local decrease of $R$ leads to a positive perturbation of $\phi$ and vice versa, which enters into Eq. (39). Hence, the nonlinearity effectively has a negative sign, which is defocusing since the sign of the coefficient in front of $R^{\prime \prime}$ in Eq. (39) is positive, and we can expect the existence of dark or gray solitons in our system.

Other special solutions (traveling wave solutions, alternative boundary conditions) could also be investigated, but we keep the above scheme, since then we can directly compare to the previous linear wave analysis. Indeed, Eqs. (37) and (39) admit the equilibrium,

$$
R^{2}=\frac{n_{0}}{\gamma}, \quad \phi=0,
$$

in the same way as the original Klein-Gordon-Maxwell system of equations (which also needs the equilibrium phase $S$ ).

To proceed, we first transform physical quantities into dimensionless variables according to

$$
\begin{aligned}
R^{*} & =\frac{R}{\sqrt{n_{0}}}, \quad \phi^{*}=\gamma+\frac{e \phi}{m c^{2}}, \quad x^{*}=\frac{\omega_{p} x}{c}, \quad p^{*}=\frac{p}{m c}, \\
S_{0}^{*} & =\frac{\omega_{p} S_{0}}{m c^{2}},
\end{aligned}
$$

so that the system for stationary waves becomes (omitting the asterisks)

$$
\begin{gathered}
H^{2} R^{\prime \prime}+\left(\phi^{2}-1\right) R=\frac{v^{2}}{R^{3}}, \\
\phi^{\prime \prime}=R^{2} \phi-1
\end{gathered}
$$

and

$$
S_{0}^{\prime}=\frac{v}{R^{2}}
$$

where $v=p / \gamma$.

In the system (42) and (43), the variable $x$ takes the role of a timelike variable, so that standard methods for ordinary differential equations can be applied. In this context, it is interesting to investigate the system around the equilibrium point if it admits only oscillatory (stable) solutions or if it also admits exponentially growing (unstable) and decaying solutions. In the latter case, there is a possibility of finding 

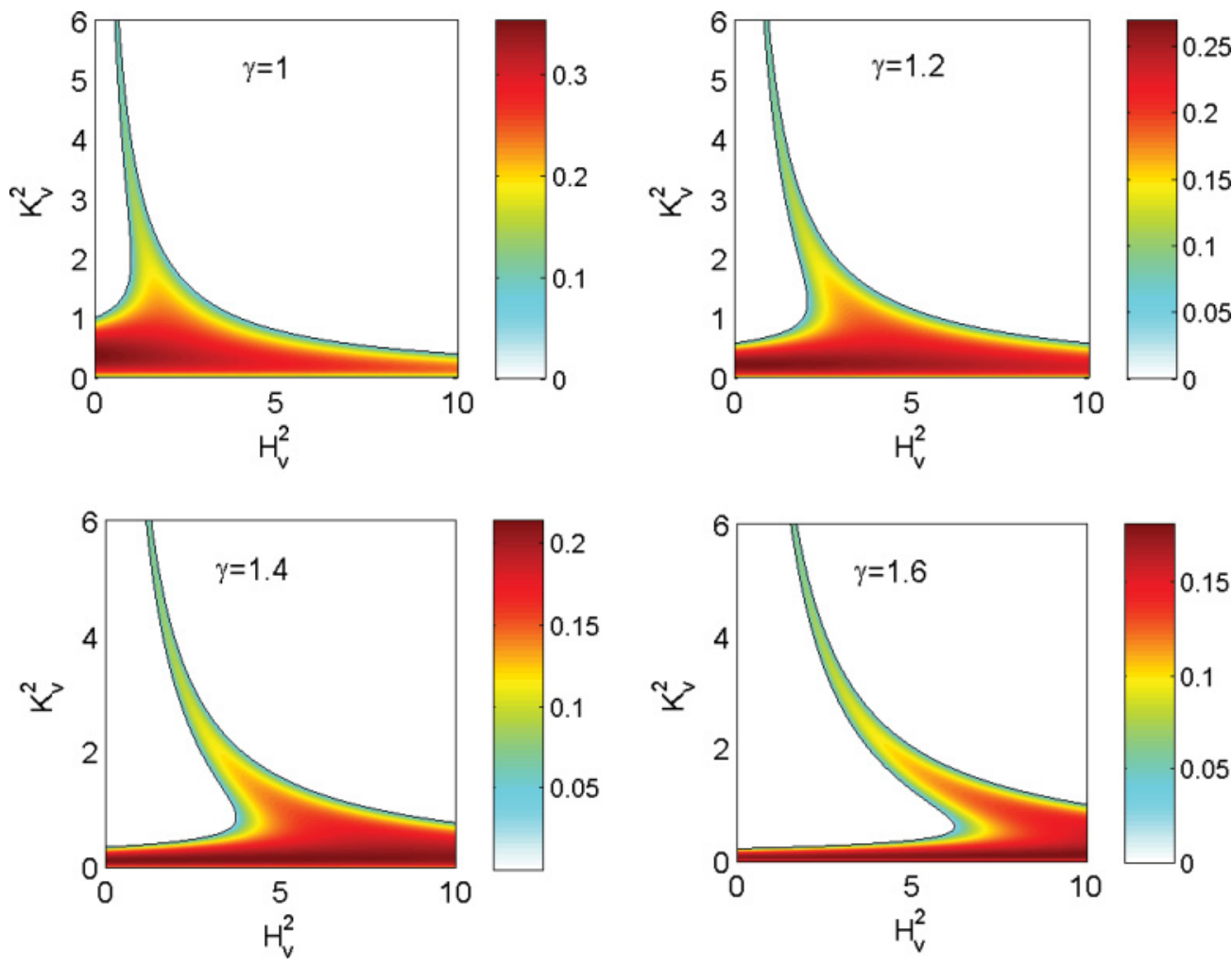

FIG. 11. (Color online) Unstable cases as a function of $H_{v}^{2}=\hbar^{2} \omega_{p}^{2} / m^{2} v^{4}$ and $K_{v}^{2}=K^{2} v^{2} / \omega_{p}^{2}$ for different values of $\gamma$, where the unstable cases, given by Eq. (82), are between the lower and upper boundaries of the colored region. The color corresponds to the normalized growth rate $\Omega_{i} / \omega_{p}$ obtained numerically from Eq. (66). The case $\gamma=1$ (upper left panel) is the nonrelativistic case corresponding to Fig. 1 of Ref. [33]. For increasing values of $\gamma$, the instability region is shifted toward larger values of $H_{v}^{2}$ and the growth rate decreases.

localized solitary wave solutions with exponentially decaying flanks, which is not possible for stable cases.

Linearizing the system around the equilibrium (40) and supposing perturbations $\propto \exp (i K x)$, we obtain the characteristic equation for the eigenvalues $K$ :

$$
\frac{1}{\gamma}\left(\frac{H^{2} K^{2}}{4}-p^{2}\right)\left(K^{2}+\frac{1}{\gamma}\right)+1=0,
$$

which is the same as Eq. (24) with $\Omega=0$. In the formal classical limit $(H=0)$, we have only linearly stable oscillations with

$$
K^{2} v^{2}=\frac{1}{\gamma^{3}}
$$

Hence, in the classical case, as is well known, we do not have localized stationary solutions. In the quantum case $(H \neq 0)$ the situation is more complex. The characteristic equation can be solved yielding $K^{2}=K_{ \pm}^{2}$, with

$$
K_{ \pm}^{2}=\frac{2}{\gamma H^{2}}\left\{\gamma p^{2}-\frac{H^{2}}{4} \pm\left[\left(\frac{H^{2}}{4}+\gamma p^{2}\right)^{2}-\gamma^{3} H^{2}\right]^{1 / 2}\right\} \text {. }
$$

Expanding for small $H$, we obtain

$$
\begin{gathered}
K_{+}^{2}=\frac{4 p^{2}}{H^{2}}-\frac{\gamma}{p^{2}}-\frac{H^{2}}{4 p^{6}}+O\left(H^{4}\right), \\
K_{-}^{2}=\frac{1}{\gamma p^{2}}+\frac{H^{2}}{4 p^{6}}+O\left(H^{4}\right) .
\end{gathered}
$$

Note that $K_{-}$is the quantum extension of the branch in Eq. (46), while $K_{+}$has no classical analog.

To investigate the stability of the equilibrium, it is useful to rewrite the characteristic equation as

$$
F\left(K^{2}\right)=\frac{\gamma}{\left(p^{2}-H^{2} K^{2} / 4\right)\left(K^{2}+1 / \gamma\right)}=1,
$$

which gives a second-degree equation for $K^{2}$. Since the characteristic function $F\left(K^{2}\right)$ satisfies

$$
F(0)=\frac{\gamma^{2}}{p^{2}} \geqslant 1, \quad F\left(K^{2}>\frac{4 p^{2}}{H^{2}}\right)<0,
$$

and has a pole at $K^{2}=4 p^{2} / H^{2}$,

$$
\lim _{K^{2} \rightarrow\left(4 p^{2} / H^{2}\right)^{\mp}} F\left(K^{2}\right)= \pm \infty,
$$

we will have stable oscillations provided that the minimum value $F_{\min }$ in the branch $0 \leqslant K^{2}<4 p^{2} / H^{2}$ satisfies

$$
F_{\min }<1 \text {. }
$$

In this case $F\left(K^{2}\right)$ intercept the value 1 at two positive $K^{2}$ values, so that the characteristic equation has only real solutions. The situation is summarized in Fig. 1.

Working out Eq. (53) we find that

$$
\gamma H^{2}<\left(p^{2}+\frac{H^{2}}{4 \gamma}\right)^{2} .
$$



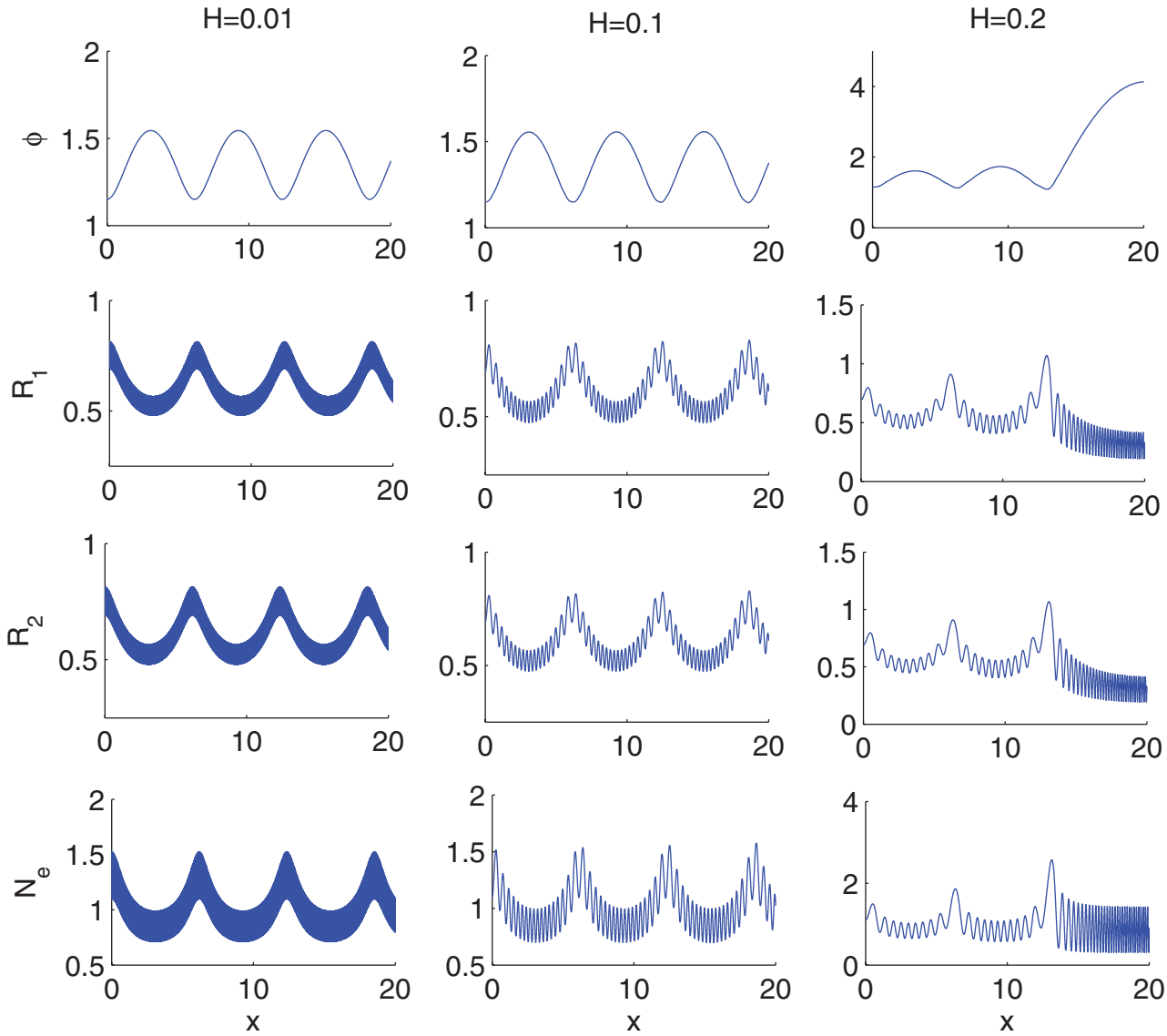

FIG. 12. (Color online) Spatial profiles of $\phi, R_{1}, R_{2}$, and the electron density $N_{e}=\left(R_{1}^{2}+R_{2}^{2}\right) \phi$ (top to bottom panels) for $H=0.01$ (left column), $H=0.1$ (middle column), and $H=0.2$ (right column). The solution was set to $\phi(0)=1.15, R_{1}(0)=R_{2}(0)=0.98 / \sqrt{2}$, and $\phi^{\prime}(0)=R_{1}^{\prime}(0)=R_{2}^{\prime}(0)=0$ at the left boundary.

Further analysis shows that the condition (54) can be written as

$$
H<H_{\max }=2 \gamma\left(\gamma^{1 / 2}-\gamma^{-1 / 2}\right),
$$

as the final condition for stable linear oscillations. It follows that the necessary existence criterion for nonlinear localized (soliton) stationary solutions is $H>H_{\max }$, while localized solutions cannot exist for $H \leqslant H_{\max }$. In Fig. 2, a graph of $H_{\max }$ is plotted as a function of the velocity $v$ (measured in units of $c$ ), which shows that the quantum range for stable oscillations is increased for increasing relativistic effects. In the nonrelativistic limit $p \ll 1$, we have $H<p^{2}=v^{2}$ as the condition for stable oscillations or, in dimensional units, $\hbar \omega_{p}<m v^{2}$.

For the nonlinear system (42) and (43), a Hamiltonian form can be obtained with the further transformation

$$
R \rightarrow i R, \quad \phi \rightarrow \frac{\phi}{H}, \quad x \rightarrow x,
$$

so that

$$
R^{\prime \prime}=-\frac{\partial V}{\partial R}, \quad \phi^{\prime \prime}=-\frac{\partial V}{\partial \phi},
$$

where

$$
V=V(R, \phi)=\frac{R^{2} \phi^{2}}{2}+\frac{\phi}{H}-\frac{R^{2}}{2 H^{2}}+\frac{v^{2}}{2 H^{2} R^{2}} .
$$

Since we arrive at an autonomous Hamiltonian system, one has the energy integral

$$
I=\frac{\left(\phi^{\prime}\right)^{2}}{2}+\frac{\left(R^{\prime}\right)^{2}}{2}+V(R, \phi) .
$$

Restoring dimensional variables, we have the conserved quantity

$$
\tilde{I}=\frac{2 \hbar^{2} \omega_{p}^{2}}{m^{2} c^{4}} I-2 \gamma
$$

or

$$
\begin{aligned}
\tilde{I}= & \frac{\hbar^{2}}{m^{2} c^{2}}\left[\left(\frac{e}{m c^{2}} \frac{d \phi}{d x}\right)^{2}-\frac{1}{n_{0}}\left(\frac{d R}{d x}\right)^{2}\right] \\
& +\frac{2 e \phi}{m c^{2}}-\frac{R^{2}}{n_{0}}\left(\gamma+\frac{e \phi}{m c^{2}}\right)^{2}+\frac{R^{2}}{n_{0}}-\frac{n_{0} \beta^{2}}{R^{2}},
\end{aligned}
$$

where $\beta=v / c$. The obtained conservation law can be used to verify the accuracy of numerical simulations.

Numerical solutions of the nonlinear system (42) and (43) are presented in Figs. 3-6. For the nonlocalized solutions in Fig. 3, initial values on $\phi$ and $R$ and their first derivatives were set on the left boundary and the solution was integrated using the standard fourth-order Runge-Kutta method. For the localized solutions in Figs. 4-6, boundary conditions on $\phi$ and $R$ were fixed on both the left and right boundaries, and 

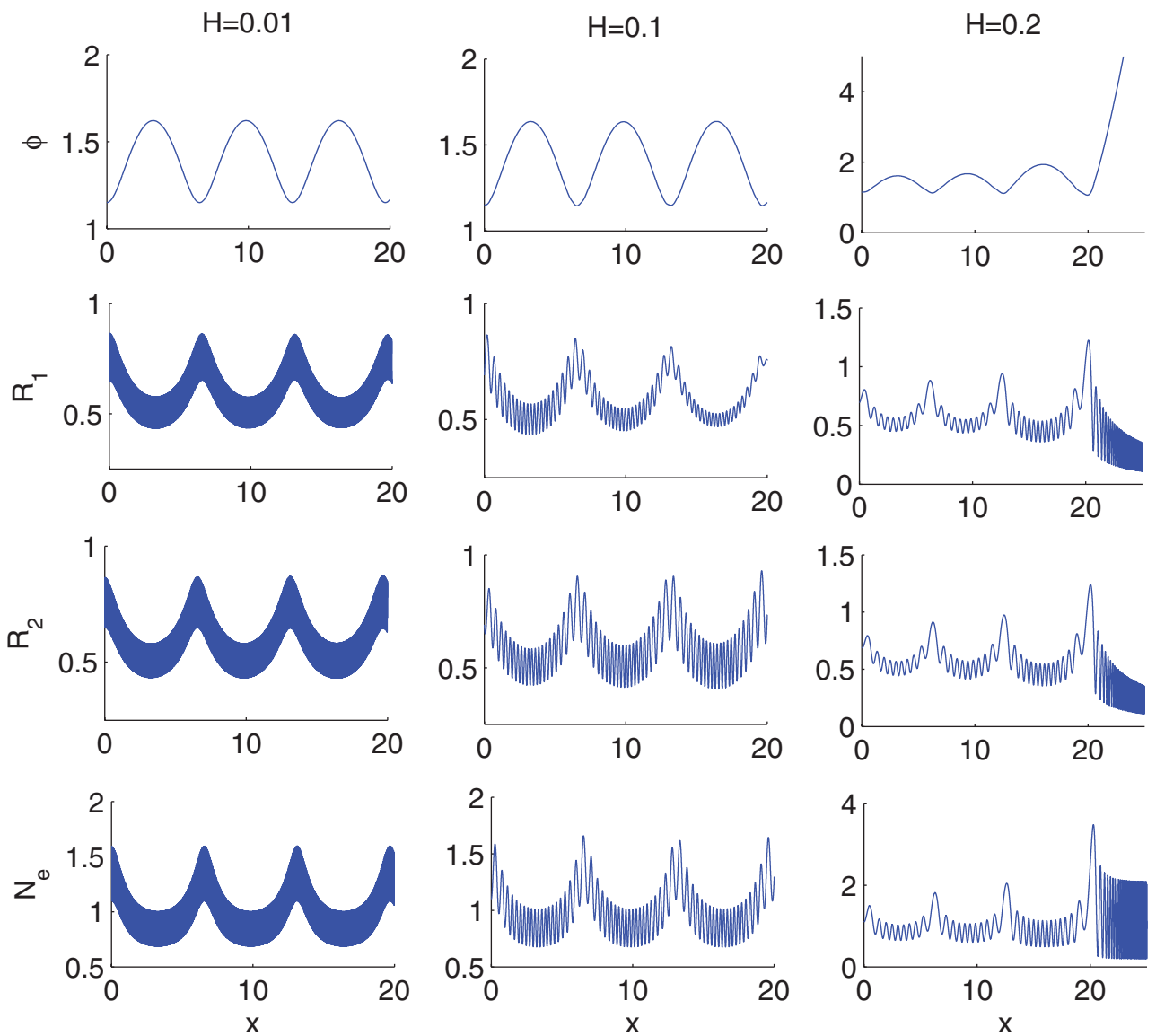

FIG. 13. (Color online) The same as in Fig. 12, but using $R_{1}^{\prime}(0)=10$ and $R_{2}^{\prime}(0)=-10$ (left column), $R_{1}^{\prime}(0)=1$ and $R_{2}^{\prime}(0)=-1$ (middle column), and $R_{1}^{\prime}(0)=0.1$ and $R_{2}^{\prime}(0)=-0.1$ (right column).

the solutions were found with iterations based on Newton's method. Figure 3 shows large amplitude oscillations for $\gamma=1.3$ and different values of $H$, such that small-amplitude oscillations are linearly stable, in the sense discussed above. We see that there is one short and one long length-scale, corresponding to $K_{+}$and $K_{-}$for the linear oscillations in Eq. (47). Here the small-scale oscillations are due to the quantum diffraction effect, while the large-scale oscillations are related to wakefield oscillations which are well known in classical plasmas [44].

For $H>H_{\max }$, where there are exponentially decaying or increasing (unstable) oscillations, we have the possibility of localized solutions in the form of dark or gray solitons. It turns out that the coupled system of Eqs. (42) and (43) supports a wide variety of nonlinear localized structures. Due to quantum diffraction effects, the plasma can develop dark solitary waves with one or more electron density minima. In Fig. 4, we see different classes of dark solitary waves for the case when the plasma is at rest, $v=0$, and $H=0.01$. Since $p=v=0$, in this case, the term proportional to $1 / R^{3}$ on the right-hand side of Eq. (42) vanishes, and $R$ can continuously go between positive and negative values. We see single, double, and triple dark solitons, where $R$ is shifted $180^{\circ}$ (from negative to positive) between the left and right sides of the single and triple dark solitons. The dark soliton with a single electron minimum is the same type as found in Ref. [45] for a nonrelativistic quantum plasma. The solutions with multiple-density minima are somewhat similar in shape to the multiple-hump optical solitons predicted in relativistic laser-plasma interactions in the classical regime $[46,47]$. On the other hand, in Figs. 5 and 6 , we consider solitons in a streaming plasma with finite speed $v>0$. We recall that the existence condition for solitons is $H>2 \gamma\left(\gamma^{1 / 2}-\gamma^{-1 / 2}\right)$ [where $\gamma=1 /\left(1-v^{2}\right)^{1 / 2}$ ], which puts an upper limit on $v$ for a given value of $H$. For example, for $H=0.01$, shown in Fig. 5, we have $v \lesssim 0.1$, while for $H=0.5$, shown in Fig. 6 , we have $v \lesssim 0.58$ for solitons to exist. For $H \ll 1$ and nonrelativistic $v \ll 1$, the existence condition for solitary structures becomes $v^{2}<H$ or, in dimensional units, $m v^{2}<\hbar \omega_{p}$. A general feature of the propagating solitons is that the electron density is nonzero at the center of the soliton; hence they are gray solitons. Furthermore, as the speed increases, the amplitudes of the solitons decrease and their tails become oscillatory when the speed approaches the maximum allowed speed, as can be seen in the right-hand columns of Figs. 5 and 6. There is also a complex phase shift proportional to $S_{0}$ in the total wave function $\psi$ due to the relation (38). The plot of $\left(S_{0}-p x\right) / H$ in Fig. 6 shows how the phase (in radians) is shifted between the two sides of the solitons. As $v \rightarrow 0$, the phase jumps abruptly a value of $\approx \pi$ in the center of the soliton, while solitons with 

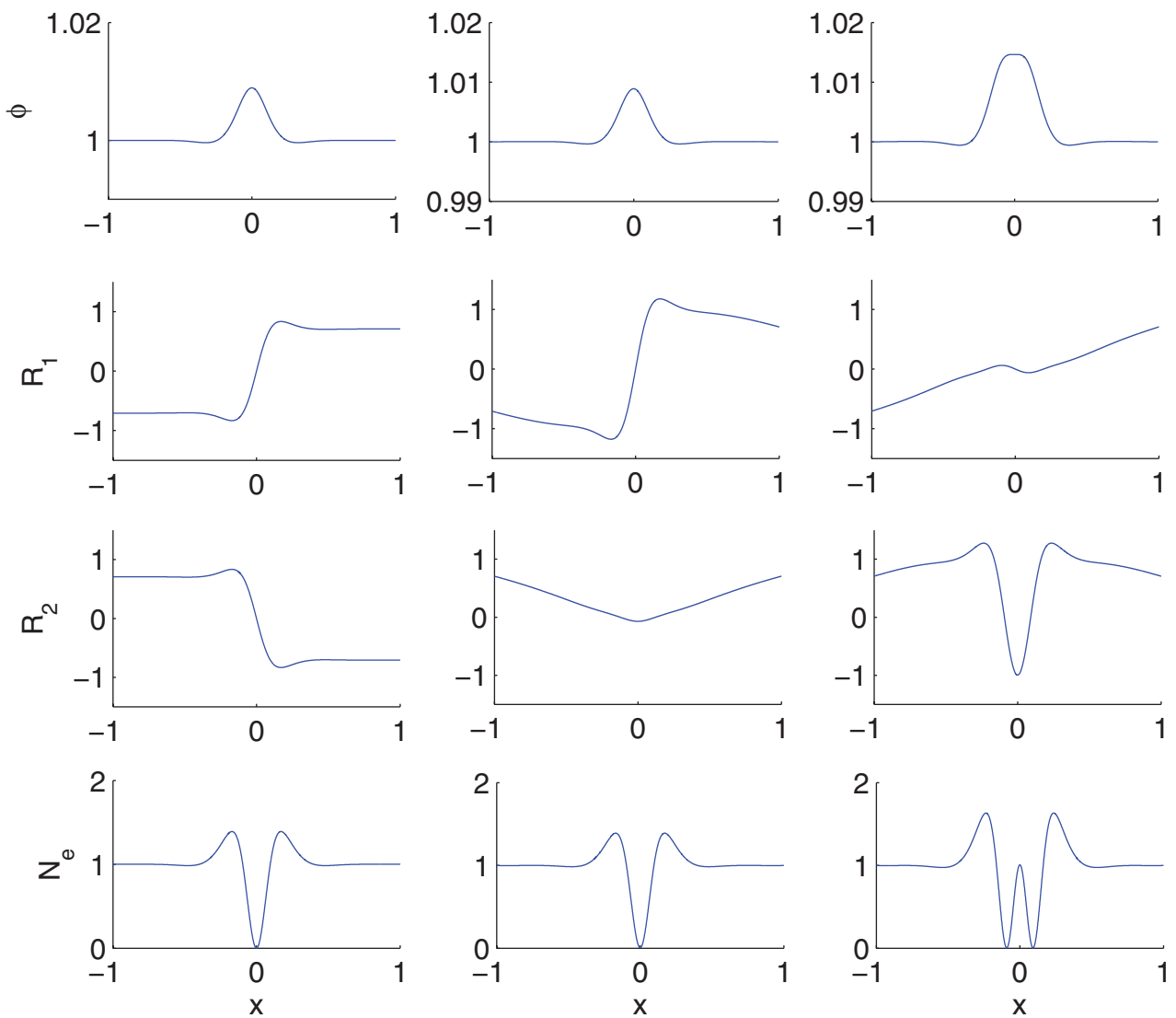

FIG. 14. (Color online) Spatial profiles of $\phi, R_{1}, R_{2}$, and $N_{e}=\left(R_{1}^{2}+R_{2}^{2}\right) \phi$ (top to bottom panels) for the zero beam speed case $v=0$ with $H=0.01$. The solution is set to $\phi=\left|R_{1}\right|=\left|R_{2}\right|=1 / \sqrt{2 \gamma}$ at the left and right boundaries.

higher speeds have smaller and smoother jumps in the phase, as can be seen in the bottom panels of Figs. 5 and 6. Likewise, magnetosonic solitary waves in spin- $1 / 2$ quantum plasmas can be also constructed [48].

\section{THE TWO-STREAM CASE}

We next consider linear and nonlinear waves for the twostream case $(N=2)$. For this case, the stream is represented by a wave function $\psi_{j}=R_{j} \exp \left(i S_{j} / \hbar\right), j=1$ and 2 , and we have the Klein-Gordon-Poisson system of equations:

$$
\begin{gathered}
\frac{1}{c^{2}}\left(\frac{\partial S_{j}}{\partial t}-e \phi\right)^{2}-\left(\nabla S_{j}\right)^{2}-m^{2} c^{2}=\frac{\hbar^{2} \square R_{j}}{R_{j}}, \\
R_{j}\left(\square S_{j}-\frac{e}{c^{2}} \frac{\partial \phi}{\partial t}\right)+\frac{2}{c^{2}} \frac{\partial R_{j}}{\partial t}\left(\frac{\partial S_{j}}{\partial t}-e \phi\right) \\
-2 \nabla R_{j} \cdot \nabla S_{j}=0, \\
\nabla^{2} \phi=-\frac{e}{\varepsilon_{0}}\left[\frac{1}{m c^{2}} \sum_{j=1}^{2} R_{j}^{2}\left(\frac{\partial S_{j}}{\partial t}-e \phi\right)+n_{0}\right],
\end{gathered}
$$

which describe a relativistic quantum two-stream plasma in the electrostatic approximation, using physical variables.

\section{A. Linear waves}

Similar to the one-stream case, we have the equilibrium

$$
\begin{aligned}
& R_{1}=R_{2}=\left(\frac{n_{0}}{2 \gamma}\right)^{1 / 2}, \quad \phi=0, \quad \gamma=\left(1+\frac{p^{2}}{m^{2} c^{2}}\right)^{1 / 2} \\
& S_{1}=-\gamma m c^{2} t+\mathbf{p} \cdot \mathbf{r}, \quad S_{2}=-\gamma m c^{2} t-\mathbf{p} \cdot \mathbf{r}
\end{aligned}
$$

for two symmetric counterpropagating electron streams.

Linearizing the governing equations and assuming planewave perturbations with the wave number $\mathbf{K} \| \mathbf{p}$ and the angular frequency $\Omega$, we obtain the dispersion relation

$$
F(\Omega)=1,
$$

with the characteristic function $F(\Omega)$ defined by

$$
F(\Omega)=\frac{\omega_{b}^{2}}{\gamma} \sum_{+,-} \frac{4 m^{2} c^{4}-\hbar^{2}\left(\Omega^{2}-c^{2} K^{2}\right)}{4 \gamma^{2} m^{2} c^{4}(\Omega \mp K v)^{2}-\hbar^{2}\left(\Omega^{2}-c^{2} K^{2}\right)^{2}},
$$

where

$$
\omega_{b}=\left(\frac{n_{0} e^{2}}{2 m \varepsilon_{0}}\right)^{1 / 2}, \quad v=p /(\gamma m) .
$$

In the classical limit, viz., $\hbar=0$, we obtain the same results as in the description of classical cold relativistic electron beams using the fluid theory [10]. It can be treated in full analytical 

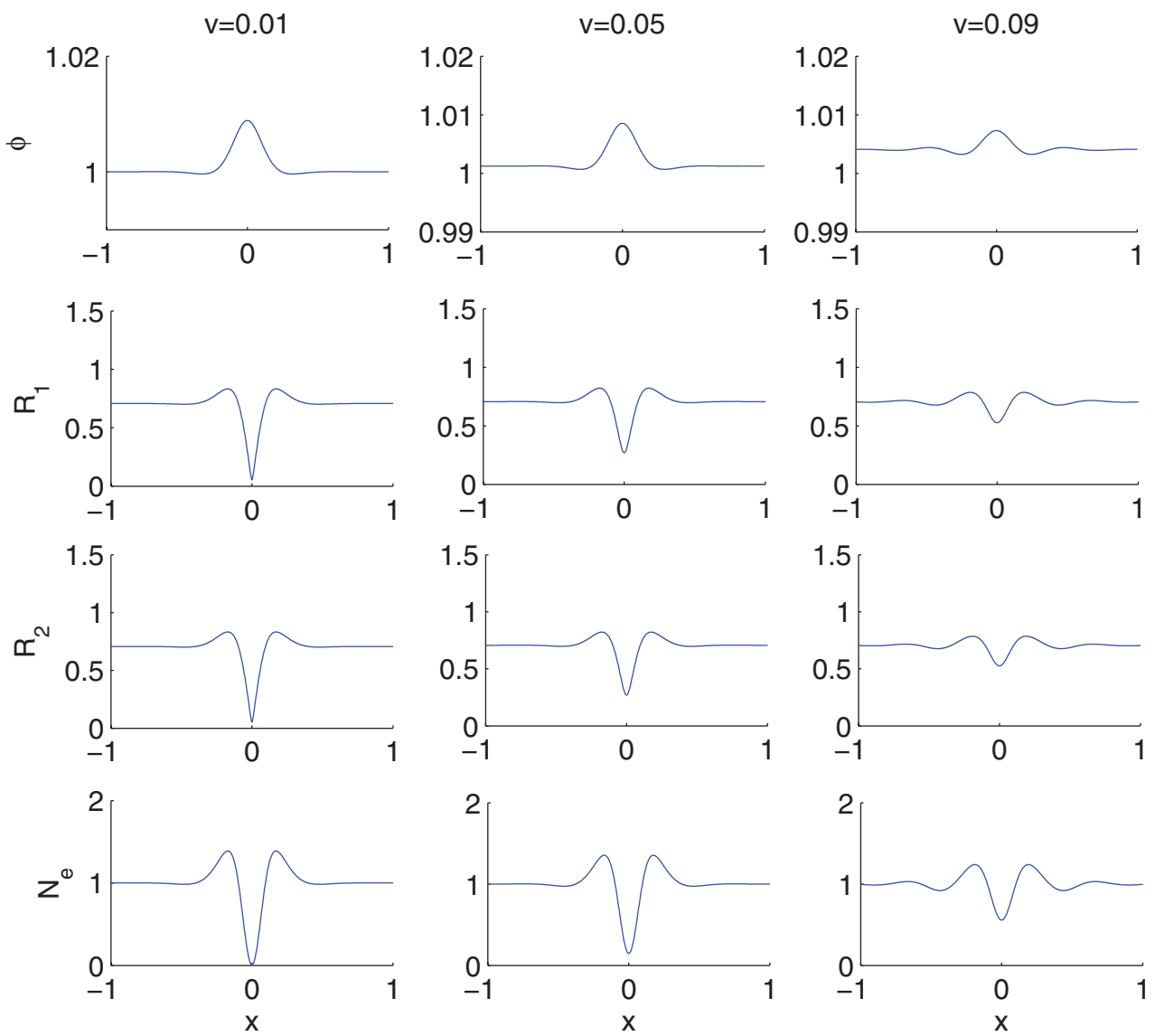

FIG. 15. (Color online) Spatial profiles of $\phi, R_{1}, R_{2}$, and $N_{e}=\left(R_{1}^{2}+R_{2}^{2}\right) \phi$ (top to bottom panels), for $H=0.01$ and $v=0.01$ (left column), $v=0.05$ (middle column), and $v=0.09$ (right column). The solution was set to $\phi=\gamma$ and $R_{1}=R_{2}=1 / \sqrt{2 \gamma}$ at the left and right boundaries. We see gray solitons with nonzero electron density in the center.

detail. We have the dispersion relation

$$
\Omega^{2}=K^{2} v^{2}+\frac{\omega_{b}^{2}}{\gamma^{3}} \pm \frac{\omega_{b}}{\gamma^{3}}\left(\omega_{b}^{2}+4 \gamma^{3} K^{2} v^{2}\right)^{1 / 2} .
$$

Equivalently, it is useful to write the classical dispersion relation as $F_{C}(\Omega)=1$, with the characteristic function

$$
F_{C}(\Omega)=\frac{\omega_{b}^{2}}{\gamma^{3}}\left[\frac{1}{(\Omega-K v)^{2}}+\frac{1}{(\Omega+K v)^{2}}\right],
$$

obtained by setting $\hbar=0$ in Eq. (67). The dispersion relation turns out to be a quadratic equation for $\Omega^{2}$; hence $F_{C}(\Omega)$ should attain the unity value four times to prevent instability. Graphically (see Fig. 7) we conclude that

$$
F_{C}(0)<1
$$

is the condition for linear stability. This shows that the wave numbers such that

$$
K^{2} v^{2}>\frac{\omega_{p}^{2}}{\gamma^{3}}
$$

are linearly stable. The same conclusion is reached when the potentially unstable mode in Eq. (69) is analyzed. In comparison with the nonrelativistic stability condition $K^{2}>$ $\omega_{p}^{2} / v^{2}$, we note that the relativistic effects are stabilizing, since they imply a smaller unstable range in wave-number space.
Setting $\Omega=i \Omega_{i}$ for real $\Omega_{i}$ and using Eq. (69), we obtain

$$
\max \left(\Omega_{i}\right)=\frac{\omega_{b}}{2 \gamma^{3 / 2}}
$$

as the maximum growth rate, which also becomes smaller due to relativistic effects.

On the other hand, in the quantum but nonrelativistic $(\hbar \neq 0$ and $c \rightarrow \infty)$ limit we have

$$
1-\sum_{+,-} \frac{\omega_{b}^{2}}{(\Omega \mp K v)^{2}-\hbar^{2} K^{4} /\left(4 m^{2}\right)}=0 .
$$

We do not discuss the nonrelativistic case, since this has been already done in the past [33]. The nonrelativistic case, as well as the nonquantum case, can be solved in full analytical detail, because in both situations the dispersion relation is equivalent to a second-degree polynomial equation for $\Omega^{2}$.

We now turn our attention to the fully quantum-relativistic dispersion relation (66). Due to the symmetry, we can restrict the treatment to positive frequencies, wave numbers, and beam velocities. Equation (66) is equivalent to a fourth-degree polynomial equation for $\Omega^{2}$, which can be analytically solved in terms of cumbersome expressions or solved numerically. However, it is more informative to first analyze the behavior of the characteristic function $F(\Omega)$ in Eq. (67), which is mainly 
determined by the poles at

$$
\begin{aligned}
& \Omega_{1}=\frac{\gamma m c^{2}}{\hbar}-\left[\left(\frac{\gamma m c^{2}}{\hbar}-K v\right)^{2}+\frac{K^{2} c^{2}}{\gamma^{2}}\right]^{1 / 2}, \\
& \Omega_{2}=-\frac{\gamma m c^{2}}{\hbar}+\left[\left(\frac{\gamma m c^{2}}{\hbar}+K v\right)^{2}+\frac{K^{2} c^{2}}{\gamma^{2}}\right]^{1 / 2}, \\
& \Omega_{3}=\frac{\gamma m c^{2}}{\hbar}+\left[\left(\frac{\gamma m c^{2}}{\hbar}-K v\right)^{2}+\frac{K^{2} c^{2}}{\gamma^{2}}\right]^{1 / 2}, \\
& \Omega_{4}=\frac{\gamma m c^{2}}{\hbar}+\left[\left(\frac{\gamma m c^{2}}{\hbar}+K v\right)^{2}+\frac{K^{2} c^{2}}{\gamma^{2}}\right]^{1 / 2},
\end{aligned}
$$

paying attention just to the positive values. More precisely, $\Omega_{1}>0$ provided $\hbar K<2 p$; otherwise the positive pole is at $-\Omega_{1}$. It can be shown that one has the ordering

$$
\left|\Omega_{1}\right|<\Omega_{2}<\Omega_{3}<\Omega_{4} .
$$

Here, $\Omega_{1}$ and $\Omega_{2}$ have classical counterparts for $\hbar \rightarrow 0$, while $\Omega_{3}$ and $\Omega_{4}$ are associated with pair branches, without classical counterparts.

We note that the case $\hbar K=2 p$ is degenerate, since then one has $\Omega_{1}=0$ and the dispersion relation becomes a third-degree polynomial equation for $\Omega^{2}$. The solutions to this particular case alway correspond to (marginally) stable modes, not considered any further here.

A tedious analysis shows that the characteristic function has the following properties:

$$
\begin{aligned}
\lim _{\Omega \rightarrow \Omega_{1}^{ \pm}} F(\Omega) & =\mp \infty, \quad \lim _{\Omega \rightarrow \Omega_{2,3,4}^{ \pm}} F(\Omega)= \pm \infty, \\
\hbar K<2 p & \Rightarrow F\left(\Omega_{1}<\Omega<\Omega_{2}\right)<0 \\
\operatorname{sgn} F(0) & =-\operatorname{sgn} F^{\prime \prime}(0)=-\operatorname{sgn}(\hbar K-2 p) .
\end{aligned}
$$

Moreover, $F(\Omega)$ tends monotonously to zero as $\Omega \rightarrow \infty$. These results imply that the wave numbers satisfying $\hbar K>$ $2 p$ are always stable, since in this case the characteristic function has the topology shown in Fig. 8, where $F(\Omega)$ always intercepts the value unity four times.

On the other hand, the case $\hbar K<2 p$ is potentially unstable, according to the minimum value $F(0)$. If $F(0)<1$, the characteristic function attains the unity value at four positive frequencies, corresponding to four linearly stable waves. Otherwise, when $F(0)>1$ there is a (purely imaginary) solution for the dispersion relation and hence instability. The whole scenario is summarized in Figs. 9 and 10.

In summary, besides $\hbar K<2 p$ we have

$$
F(0)=\frac{\omega_{p}^{2}}{\gamma^{3} K^{2} v^{2}} \frac{1+\hbar^{2} K^{2} /\left(4 m^{2} c^{2}\right)}{1-\hbar^{2} K^{2} /\left(4 p^{2}\right)}>1
$$

as a necessary condition for unstable linear wave propagation in our two-stream relativistic quantum plasma. Rearranging the instability conditions found, we combine them according to

$$
4 p^{2}>\hbar^{2} K^{2}>4\left(\frac{\gamma K^{2} v^{2} / \omega_{p}^{2}-1+\beta^{2}}{\gamma K^{2} v^{2} / \omega_{p}^{2}+\beta^{2}}\right) p^{2}
$$

It can be verified that Eq. (82) reproduces the nonrelativistic results [33].
The instability condition (82), together with a numerical solution of Eq. (66), is depicted in Fig. 11. Here we assumed that $\Omega=\Omega_{r}+i \Omega_{i}$, where $\Omega_{r}$ is the real frequency and $\Omega_{i}$ is the growth rate, and plotted $\Omega_{i} / \omega_{p}$ as a function of $H_{v}^{2}=\hbar^{2} \omega_{p}^{2} / m^{2} v^{4}$ and $K_{v}^{2}=K^{2} v^{2} / \omega_{p}^{2}$ for different values of $\gamma$. The case $\gamma=1$ corresponds to Fig. 1 of Ref. [33], while $\gamma>1$ shows the relativistic effects on the instability region. We note that in the formal classical limit the largest unstable wave number becomes smaller as $\gamma \rightarrow \infty$. On the other hand, the height of the upper curve in the instability diagram scales as $\gamma^{2}$, so that in this sense the combined quantum-relativistic effects tend to enlarge the unstable area. Ultimately, however, quantum effects stabilize the sufficiently small wave numbers, no matter the strength of the relativistic effects. An interesting quantum effect is the appearance of an instability region at large wave numbers $K_{v}^{2}=K^{2} v^{2} / \omega_{p}^{2}$ for moderately small values of $H_{v}^{2}=\hbar^{2} \omega_{p}^{2} / m^{2} v^{4}$, which does not have a classical counterpart. Finally, it should be noted that simultaneously $H_{v}^{2} \gtrsim 1$ and $\gamma>1$ in Fig. 11 correspond to extremely high electron number densities, comparable to those in the interiors of white dwarf stars and similar astrophysical objects.

\section{B. Nonlinear stationary solutions}

We consider the one-dimensional version of the system (62)-(64) and stationary solutions of the forms

$$
R_{1,2}=R_{1,2}(x), \quad S_{1,2}=-\gamma m c^{2} t+\sigma_{1,2}(x), \quad \phi=\phi(x) .
$$

Equation (63) is then equivalent to

$$
\frac{d}{d x}\left(R_{1}^{2} \sigma_{1}^{\prime}\right)=\frac{d}{d x}\left(R_{2}^{2} \sigma_{2}^{\prime}\right)=0
$$

where the primes denote $x$ derivatives. Assuming

$$
R_{1}^{2}=R_{2}^{2}=\frac{n_{0}}{2 \gamma} \quad \text { and } \quad \sigma_{1}^{\prime}=-\sigma_{2}^{\prime}=\mathrm{p}
$$

at equilibrium, applying the transformation

$$
R_{1,2}^{*}=\frac{R_{1,2}}{\sqrt{n_{0}}}, \quad \phi^{*}=\gamma+\frac{e \phi}{m c^{2}}, \quad x^{*}=\frac{\omega_{b} x}{c},
$$

and using Eq. (84) to eliminate $\sigma_{1,2}^{\prime}$ from Eq. (62), we readily derive the system of equations (omitting the asterisks)

$$
\begin{aligned}
& H^{2} R_{1}^{\prime \prime}+\left(\phi^{2}-1\right) R_{1}=\frac{v^{2}}{4 R_{1}^{3}}, \\
& H^{2} R_{2}^{\prime \prime}+\left(\phi^{2}-1\right) R_{2}=\frac{v^{2}}{4 R_{2}^{3}},
\end{aligned}
$$

and

$$
\phi^{\prime \prime}=\left(R_{1}^{2}+R_{2}^{2}\right) \phi-1,
$$

which predict nonlinear stationary solutions of a relativistic quantum two-stream plasma. Here $H$ and $v$ are defined as in the one-stream case.

Linearizing around $R_{1,2}^{2}=1 /(2 \gamma)$ and $\phi=\gamma$, and supposing perturbations $\propto \exp (i K x)$, we obtain a quadratic equation for $K^{2}$, which is also obtained from Eq. (66) when setting $\Omega=0$. Proceeding as before, we formally obtain the same existence condition as Eq. (55) for periodic solutions. The 
two-stream nonlinear solutions can be constructed from the one-stream cases by using $\left|R_{1}\right|=\left|R_{2}\right|=|R| / \sqrt{2}$, where $R$ is obtained by solving the system (42) and (43). The signs of $R_{1}$ and $R_{2}$ are arbitrary. In Fig. 12, we show a numerical solution of the system (87)-(89), where the profiles of $\phi$ and $N_{e}$ are identical to the ones in Fig. 3 and with $R_{1}=R_{2}=R / \sqrt{2}$. In Fig. 13, we perturbed this solution by using different values of $R_{1}^{\prime}(0)$ and $R_{2}^{\prime}(0)$ at the left boundary. The general behavior of the solution in Fig. 13 remains similar to that in Fig. 12, but the differences in the two solutions can be seen in the details.

Stationary localized solutions are shown in Figs. 14 and 15 for the nonstreaming $(v=0)$ and streaming $(v>0)$ cases, respectively. In both cases, we found only localized solutions corresponding to $\left|R_{1}\right|=\left|R_{2}\right|=|R| / \sqrt{2}$ (where $R$ is the onestream solution), but no other, more complicated cases. For the nonstreaming solutions in Fig. 14 we show an example with $\left|R_{1}\right|=\left|R_{2}\right|=|R| / \sqrt{2}$ in the first column (with opposite signs on $R_{1}$ and $R_{2}$ ). When the solution was forced to an antisymmetric $R_{1}$ and a symmetric $R_{2}$ in space, the numerical solution converged to solutions where either $R_{1}$ or $R_{2}$ took the shape of a one-stream dark soliton, while the other part tended to zero (or as small as possible) close to the soliton. For the streaming case in Fig. 15, we also only found localized solutions corresponding to $\left|R_{1}\right|=\left|R_{2}\right|=|R| / \sqrt{2}$. Similar to the one-stream case, we have a maximum beam speed of $v \approx 0.1$ for the existence of localized solutions, and as the beam speed approaches this value, the amplitude of the soliton decreases and becomes oscillatory in space.

One further issue is the stability of the localized solutions in the streaming cases $v>0$. Far away from the localized solution, the plasma can be considered to be homogeneous, and one can perturb the equilibrium and study plane-wave solutions proportional to $\exp (-i \Omega t+i K x)$ for real-valued $K$ and complex-valued $\Omega$ with unstable solutions if the imaginary part of $\Omega$ is positive. In the one-stream case, studied in Sec. II, all solutions were found to be stable in time, while in the two-stream case, studied in Sec. IV, we have $K$ for which the solutions are unstable. Hence, for the two-stream case the system is sensitive to perturbations far away from the localized structure. The general stability analysis for localized solutions can be carried out with normal mode analysis by perturbing the nonlinear equilibrium solution of the system (62)-(64) as $R_{1,2}(x, t)=R_{1,2}(x)+\widehat{R}_{1}(x) \exp (-i \Omega t)$, $S_{1,2}(x, t)=-\gamma m c^{2} t+\sigma_{1,2}(x)+\widehat{S}_{1,2}(x) \exp (-i \Omega t)$, and $\phi(x, t)=\phi(x)+\widehat{\phi}(x) \exp (-i \Omega t)$, and assuming that the perturbed quantities vanish at $|x|=\infty$. This leads to a linear eigenvalue problem with eigenfunctions $\widehat{R}_{1}(x), \widehat{S}_{1,2}(x)$, and $\widehat{\phi}(x)$ and eigenvalue $\Omega$. Solutions with $\Omega$ having positive imaginary parts are unstable and will grow exponentially with time.

\section{SUMMARY AND CONCLUSION}

In this paper, we have presented a multistream model for a relativistic quantum plasma, using the Klein-Gordon model for the electrons. We have treated the one- and two-stream cases in detail. We have derived dispersion relations for the linear beam-plasma interactions in the one-stream case and for the streaming instability in the two-stream case. The system exhibits both plasma oscillations close to the plasma wave frequency that reduce to the Langmuir oscillation frequency in the classical limit $\hbar \rightarrow 0$ and pair branches which do not have a classical analog. Also, there is a new instability branch for large wave numbers of pure quantum origin. A main result of this work is the derivation of the instability condition in Eq. (82), which provides a natural generalization to the nonrelativistic instability criterion [33]. Another important result is the condition (55) for the existence of periodic (stable) oscillations and exponentially growing and decaying (unstable) steady-state oscillations, where the latter exist only below a given electron beam speed. Similar to the classical plasma case [49], this furnishes an existence condition for periodic solutions, which exists only for cases with exponentially growing and decaying solutions. A rich variety of nonlinear solutions has been numerically found, including solitary waves with one or more electron density minima and an associated positive potential. It has been noted that the amplitude of the solitons decreased for increasing beam speeds, with increasingly oscillatory tails as the beam speed approached its maximum value for the existence of soliton solutions.

Our model can be applied to situations where the quantum statistical thermal and electron degeneracy pressure effects are small. The relative importance of these two effects can be characterized by the degeneracy parameter $\chi=T_{F} / T$, where $T_{F}=\hbar^{2}\left(3 \pi^{2} n_{0}\right)^{2 / 3} /\left(2 \kappa_{B} m\right)$ is the Fermi electron temperature and $\kappa_{B}$ is the Boltzmann constant. When $\chi<1$, the thermal pressure dominates, while when $\chi>1$, the degeneracy pressure dominates. Our model is applicable when $\kappa_{B} T \ll m v^{2}$ for $\chi>1$ and $\kappa_{B} T_{F} \ll m v^{2}$ for $\chi<1$. Strong coupling (collisional) effects can be neglected when the coupling constant $\Gamma=e^{2} n_{0}^{1 / 3} /\left(4 \pi \varepsilon_{0} \kappa_{B} T\right.$ ) (for $\chi<1$ ) or $\Gamma=e^{2} n_{0}^{1 / 3} /\left(4 \pi \varepsilon_{0} \kappa_{B} T_{F}\right)$ (for $\left.\chi>1\right)$ is small. For $\chi>1$, the Pauli blocking further helps to reduce the effect of collisions [50]. The pair creation phenomena have been neglected here, because our model excludes quantized fields. Hence, $\hbar \omega_{p}$ needs to be much smaller than $2 m c^{2}$ [43]. Working out the weak coupling and no quantized field assumptions, formulated as $\Gamma<1$ and $\hbar \omega_{p} /\left(2 m c^{2}\right)<1$, we have (using SI units)

$$
\begin{gathered}
\log _{10} T>\frac{1}{3} \log _{10} n_{0}-4.8, \quad \text { if } \quad \chi<1, \\
\log _{10} n_{0}>28.8, \quad \text { if } \quad \chi>1,
\end{gathered}
$$

and

$$
\log _{10} n_{0}<38.9
$$

as the condition for the applicability of our theoretical model.

Furthermore, our model is not applicable to strong electric fields approaching the Schwinger field $\sim m^{2} c^{3} /(e \hbar)$ where electron-positron pairs are predicted to be created from vacuum polarization [51]. Thus we are restricted to $|\partial \phi / \partial x|<m^{2} c^{3} /(e \hbar)$ (in our normalized variables $|\partial \phi / \partial x|<$ $1 / H)$, which puts a limit on the wave amplitude in the model.

Finally, it should be noted that our investigation neglects electron spin-1/2 effects, which are justified since we used an unmagnetized quantum plasma model, and hence there are no spin couplings to a magnetic field. Also, spin-spin interactions were not taken into account. In conclusion, we 
stress that the present investigation of linear and nonlinear effects dealing with relativistic electron beams in a quantum plasma is relevant for high intensity laser-plasma interaction experiments [1], white dwarf stars [52,53], and neutron stars [12-14], where both quantum and relativistic effects could be important. The multistream model can also be useful for studying relativistic interactions between electromagnetic waves and electron beams with applications to quantum free electron lasers [54,55], where the energy and momentum spread of the electrons (the beam emittance) may influence the laser gain.

\section{ACKNOWLEDGMENTS}

This work was supported by the Conselho Nacional de Desenvolvimento Científico e Tecnológico ( $\mathrm{CNPq}$ ) as well as by the Deutsche Forschungsgemeinschaft through Project SH21/3-2 of Research Unit 1048.
[1] G. A. Mourou, T. Tajima, and S. V. Bulanov, Rev. Mod. Phys. 78, 309 (2006); M. Marklund and P. K. Shukla, ibid. 78, 591 (2006).

[2] D. B. Melrose and A. Mushtaq, Phys. Plasmas 17, 122103 (2010).

[3] A. Mushtaq and D. B. Melrose, Phys. Plasmas 16, 102110 (2009).

[4] R. Hakim and J. Heyvaerts, Phys. Rev. A 18, 1250 (1978); H. D. Sivak, ibid. 34, 653 (1986).

[5] J. T. Mendonça, Phys. Plasmas 18, 062101 (2011).

[6] B. Eliasson and P. K. Shukla, Phys. Rev. E 83, 046407 (2011).

[7] B. Eliasson and P. K. Shukla, Phys. Rev. E 84, 036401 (2011).

[8] D. B. Melrose and A. Mushtaq, Phys. Rev. E 82, 056402 (2010).

[9] D. B. Melrose and A. Mushtaq, Phys. Rev. E 83, 056404 (2011).

[10] L. E. Thode and R. N. Sudan, Phys. Rev. Lett. 30, 732 (1973); Phys. Fluids 18, 1552 (1975).

[11] E. Nakar, A. Bret, and M. Milosavljević, Astrophys. J. 738, 93 (2011).

[12] N. Andersson, G. L. Comer, and R. Prix, Phys. Rev. Lett. 90, 091101 (2003).

[13] N. Andersson, G. L. Comer, and R. Prix, Mon. Not. R. Astron. Soc. 354, 101 (2004).

[14] L. Samuelsson, C. S. Lopez-Monsalvo, N. Andersson, and G. L. Comer, Gen. Relativ. Gravit. 42, 413 (2010).

[15] L. Stenflo, Plasma Phys. 10, 551 (1968).

[16] B. B. Robinson and G. A. Swartz, J. Appl. Phys. 38, 2461 (1967).

[17] Z. S. Gribnikov, N. Z. Vagidov, and V. V. Mitin, J. Appl. Phys. 88, 6736 (2000).

[18] P. K. Shukla and B. Eliasson, Phys.-Usp. 53, 51 (2010).

[19] P. K. Shukla and B. Eliasson, Rev. Mod. Phys. 83, 885 (2011).

[20] S. V. Vladimirov and Yu. O. Tsyshetskiy, Phys.-Usp. 54, 1243 (2011).

[21] F. Haas, Quantum Plasmas: An Hydrodynamic Approach (Springer, New York, 2011).

[22] T. Takabayasi, Prog. Theor. Phys. 13, 222 (1955); Phys. Rev. 102, 297 (1956); Nuovo Cimento 3, 233 (1956); Prog. Theor. Phys. Suppl. 4, 2 (1957).

[23] F. A. Asenjo, V. Muñoz, J. A. Valdivia et al., Phys. Plasmas 18, 012107 (2011).

[24] J. Zhu and P. Ji, Phys. Rev. E 81, 036406 (2010).

[25] W. Masood, B. Eliasson, and P. K. Shukla, Phys. Rev. E 81, 066401 (2010).

[26] G. Brodin and M. Marklund, New J. Phys. 9, 277 (2007); G. Brodin et al., ibid. 13, 083017 (2011).

[27] M. Marklund and G. Brodin, Phys. Rev. Lett. 98, 025001 (2007). [28] P. K. Shukla, Nat. Phys. 5, 92 (2009).
[29] J. Lundin and G. Brodin, Phys. Rev. E 82, 056407 (2010).

[30] T. Takabayasi, Prog. Theor. Phys. 9, 187 (1953).

[31] A. V. Andreev, Laser Phys. 13, 1536 (2003).

[32] J. Dawson, Phys. Fluids 4, 869 (1961).

[33] F. Haas, G. Manfredi, and M. R. Feix, Phys. Rev. E 62, 2763 (2000).

[34] G. Brodin and M. Marklund, Phys. Rev. E 76, 055403 (2007).

[35] F. Haas, A. Bret, and P. K. Shukla, Phys. Rev. E 80, 066407 (2009); F. Haas and A. Bret, Europhys. Lett. 97, 26001 (2012); D. Anderson, B. Hall, M. Lisak, and M. Marklund, Phys. Rev. E 65, 046417 (2002).

[36] H. Feshbach and F. Villars, Rev. Mod. Phys. 30, 24 (1958).

[37] V. Kowalenko, N. E. Frankel, and K. C. Hines, Phys. Rep. 126, 109 (1985).

[38] B. B. Godfrey, W. R. Shanahan, and L. E. Thode, Phys. Fluids 18, 346 (1975).

[39] Y. T. Yan and J. M. Dawson, Phys. Rev. Lett. 57, 1599 (1986).

[40] A. Serbeto, L. F. Monteiro, K. H. Tsui, and J. T. Mendonça, Plasma Phys. Controlled Fusion 51, 124024 (2009).

[41] H. Azechi et al., Laser Part. Beams 9, 193 (1991).

[42] R. Kodama et al., Nature (London) 412, 798 (2001).

[43] V. N. Tsytovich, Sov. Phys. JETP 13, 1249 (1961).

[44] V. I. Berezhiani and I. G. Murusidze, Phys. Lett. A 148, 338 (1999).

[45] P. K. Shukla and B. Eliasson, Phys. Rev. Lett. 96, 245001 (2006); 99, 096401 (2007).

[46] P. K. Kaw, A. Sen, and T. Katsouleas, Phys. Rev. Lett. 68, 3172 (1992).

[47] V. Saxena, A. Das, A. Sen, and P. Kaw, Phys. Plasmas 13, 032309 (2006).

[48] A. Mushtaq and S. V. Vladimirov, Phys. Plasmas 17, 102310 (2010); Eur. Phys. J. D 64, 419 (2011).

[49] J. F. McKenzie and T. B. Doyle, Phys. Plasmas 9, 55 (2002).

[50] N. W. Ashcroft and N. D. Mermin, Solid State Physics (Saunders College Publishing, Orlando, 1976).

[51] J. Schwinger, Phys. Rev. 82, 664 (1951).

[52] D. K. Galloway and J. L. Sokoloski, Astrophys. J. 613, L61 (2004).

[53] D. E. Winget and S. O. Kepler, Annu. Rev. Astron. Astrophys. 46, 157 (2008).

[54] N. Piovella, M. M. Cola, L. Volpe, A. Schiavi, and R. Bonifacio, Phys. Rev. Lett. 100, 044801 (2008).

[55] B. Eliasson and P. K. Shukla, e-print arXiv:1112.5558. 